\title{
Mcl-1 is a key regulator of the ovarian reserve
}

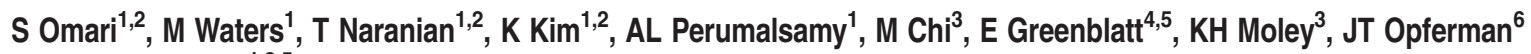 \\ and A Jurisicova ${ }^{*, 1,2,5}$
}

A majority of ovarian follicles are lost to natural death, but the disruption of factors involved in maintenance of the oocyte pool results in a further untimely follicular depletion known as premature ovarian failure. The anti-apoptotic B-cell lymphoma 2 (Bcl-2) family member myeloid cell leukemia-1 (MCL-1) has a pro-survival role in various cell types; however, its contribution to oocyte survival is unconfirmed. We present a phenotypic characterization of oocytes deficient in $\mathbf{M c l}-1$, and establish its role in maintenance of the primordial follicle (PMF) pool, growing oocyte survival and oocyte quality. Mcl-1 depletion resulted in the premature exhaustion of the ovarian reserve, characterized by early PMF loss because of activation of apoptosis. The increasingly diminished surviving cohort of growing oocytes displayed elevated markers of autophagy and mitochondrial dysfunction. Mcl-1deficient ovulated oocytes demonstrated an increased susceptibility to cellular fragmentation with activation of the apoptotic cascade. Concomitant deletion of the pro-apoptotic Bcl-2 member Bcl-2-associated X protein (Bax) rescued the PMF phenotype and ovulated oocyte death, but did not prevent the mitochondrial dysfunction associated with Mcl-1 deficiency and could not rescue long-term breeding performance. We thus recognize MCL-1 as the essential survival factor required for conservation of the postnatal PMF pool, growing follicle survival and effective oocyte mitochondrial function.

Cell Death and Disease (2015) 6, e1755; doi:10.1038/cddis.2015.95; published online 7 May 2015

Estimates of the human primordial follicle (PMF) reservoir, the size of which dictates the extent of the ovarian reserve, indicates the presence of at least half a million oocytes per ovary at birth. ${ }^{1,2}$ The essential decision that PMFs face is either long-term arrest with a possibility of recruitment toward the growing pool, or death. Even upon recruitment to the growing pool, intricately orchestrated crosstalk of survival signals between ovarian somatic cells and oocytes facilitate the ovulation of a single oocyte in human in each cycle. Hence, the default fate for millions of ovarian germ cells is death, as only a small fraction survive till ovulation. ${ }^{3}$ Insufficient endowment during fetal development or excessive oocyte loss during postnatal life further limits the ovarian reserve and can result in an untimely exhaustion of the follicle pool leading to premature ovarian failure (POF); a syndrome that affects around $1 \%$ of all women, with a higher prevalence (up to $30 \%$ ) in families with heritable traits of this condition. ${ }^{4,5}$ Mechanisms responsible for maintenance of the follicular reserve are poorly understood, however, biological assessments and mathematical modeling reveal that progressive loss of follicles with age is non-linear and accelerates, especially after 38 years. ${ }^{6,7}$ With a declining ovarian reserve, poor oocyte quality is an additional factor that contributes to the reduced fertility associated with increased maternal age. Oocytes and resulting embryos of older mothers have increased rates of aneuploidies likely due to defects in chromosomal cohesion and meiotic spindle stability, decreased DNA repair capacity, altered gene expression, impaired mitochondrial function and elevated cellular redox, all contributing to increased rates of cell death. ${ }^{8-10}$

The marked decline of oocyte number in mammalian ovaries has been attributed to oocyte loss via stage-specific modes of death. As yet, perinatal PMF loss in mice most frequently engages apoptotic cell death, ${ }^{11,12}$ whereas within the postnatal ovary, oocytes in growing follicles undergo atresia, a less 'molecularly' defined death, carrying hallmarks of both apoptosis and autophagy. ${ }^{13-15}$ It is thus surprising that no member of the anti-apoptotic B-cell lymphoma 2 (Bcl-2) family has been identified with a definitive role in governing oocyte survival and the maintenance of the ovarian reserve. $\mathrm{BCl}-2 \mathrm{l} / \mathrm{BCl}-\mathrm{w}$ and $\mathrm{BCl}-2-1 / 10 /$ Diva deficiency had no apparent impact on the ovarian reserve, and although ablation of $\mathrm{BCl}-2$ led to a loss of one-third of the adult PMF pool, the growing follicle pool was not significantly impacted and these animals did not undergo POF. ${ }^{16-19}$ Conditional Bcl-x (Bcl-2/1) inactivation led to increased primordial germ cell apoptosis in the

\footnotetext{
${ }^{1}$ Lunenfeld Tanenbaum Research Institute, Mount Sinai Hospital, 25 Orde Street, Toronto, Ontario M5T 3H7, Canada; ${ }^{2}$ Department of Physiology, University of Toronto, 1 King's College Circle, Toronto, Ontario M5S 1A8, Canada; ${ }^{3}$ Department of Obstetrics and Gynecology, Washington University in St. Louis, 660S Euclid Avenue, St. Louis, MO 63110, USA; ${ }^{4}$ Centre for Fertility and Reproductive Health, Mount Sinai Hospital, 250 Dundas Street, Toronto, Ontario M5T 2Z5, Canada; ${ }^{5}$ Department of Obstetrics and Gynecology, University of Toronto, 92 College Street, Toronto, Ontario M5G 1L4, Canada and ${ }^{6}$ Department of Biochemistry, St. Jude Children's Research Hospital, MS 340 , Room D4063D, 262 Danny Thomas Place, Memphis, TN 38105, USA

${ }^{*}$ Corresponding author: A Jurisicova, Lunenfeld Tanenbaum Research Institute, Mount Sinai Hospital, 25 Orde Street, Room 6-1016-1, Toronto, ON M5T 3H7, Canada. Tel: +1 416586 4800x2052; Fax: +1 416586 5993; E-mail: jurisicova@lunenfeld.ca

Abbreviations: AIF, apoptosis-inducing factor; Bax, Bcl-2-associated X protein; Bcl-2, B-cell lymphoma 2; BaxKO, Bax knockout; GV, germinal vesicle; LAMP, Iysosomeassociated membrane protein; MAP1LC3A, microtubule-associated protein 1 light chain; Mcl-1, myeloid cell leukemia-1; Mcl-1cKO, Mcl-1 conditional knockout; Mcl-1c/ BaxDKO, Double knockout with conditional Mcl-1 and total Bax deletion; MII, metaphase II; PA, preantral; PCD, programmed cell death; PF, primary follicle; PMF, primordial follicle; PMSG, pregnant mare's serum gonadotropin; PN, postnatal day; POF, premature ovarian failure; ROS, reactive oxygen species; SF, secondary follicle; TUNEL, Terminal deoxynucleotidyl transferase dUTP nick end labeling; Z/AP, lacZ/alkaline phosphatase reporter; ZP3, zona pellucida 3

Received 14.11.14; revised 07.2.15; accepted 18.2.15; Edited by E Baehrecke
} 
embryo, ${ }^{20}$ but postnatal inactivation of $\mathrm{Bcl}-x$ in oocytes did not compromise the ovarian reserve in young females. ${ }^{21} \mathrm{Bcl} 2 \mathrm{a} 1 \mathrm{al}$ $B f l-1 / A 1$ was low to undetectable in fully grown germinal vesicle (GV) or ovulated murine oocytes, ${ }^{22}$ however, the impact of Bfl-1 deficiency on the ovarian reserve has not yet been analyzed to the best of our knowledge. Consequently, either various anti-apoptotic $\mathrm{Bcl}-2$ members have overlapping roles in governing postnatal oocyte survival and maintenance of the adult ovarian reserve in mice, or the anti-apoptotic Bcl-2 member that regulates this decision has yet to be identified.

\section{Results}

Myeloid cell leukemia-1 (MCL-1) in oocytes is reduced in association with aging and activation of follicle atresia. With the majority (99\%) of all oocytes undergoing some form of programmed cell death (PCD), ${ }^{3,13-15,23}$ and only a few Bcl-2 family members identified with roles in regulation of germ cell fate, ${ }^{16-21}$ we set to investigate which members of the anti-apoptotic Bcl-2 gene family decline with maternal age in human GV stage oocytes. A reduction in age-dependent oocyte quality has been previously associated with activation of cell death or molecular pathways that regulate this fate. ${ }^{8,10}$ From the analyzed targets (BCL-2, BCL-2L1, BCL-2L10, $B C L-2 L 11$ and $M C L-1)$, only the $M C L-1$ transcript significantly declined in an age-associated manner, with a decrease in MCL-1 immunoreactivity in human GV oocytes (Figure 1a). Changes in MCL-1 transcript associated with differences in clinical diagnoses, stimulation protocol or resultant outcome proved nonsignificant (Supplementary Table S2). MCL-1 has been shown to be expressed by fetal human and neonatal mouse oocytes, ${ }^{16,24}$ and abundant transcript levels were detected in ovulated human oocytes. ${ }^{25}$ We assessed the expression of MCL-1 in murine ovaries and before pubertal onset cytoplasmic immunoreactivity of MCL-1 in oocytes increased with activation of follicle growth (from PMF to primary follicle (PF)), remained robust in the fully grown oocytes of preantral (PA) follicles and virtually disappeared from oocytes undergoing atresia (Figure 1b). This pattern indicated that MCL-1 could be an essential regulator of oocyte survival. a

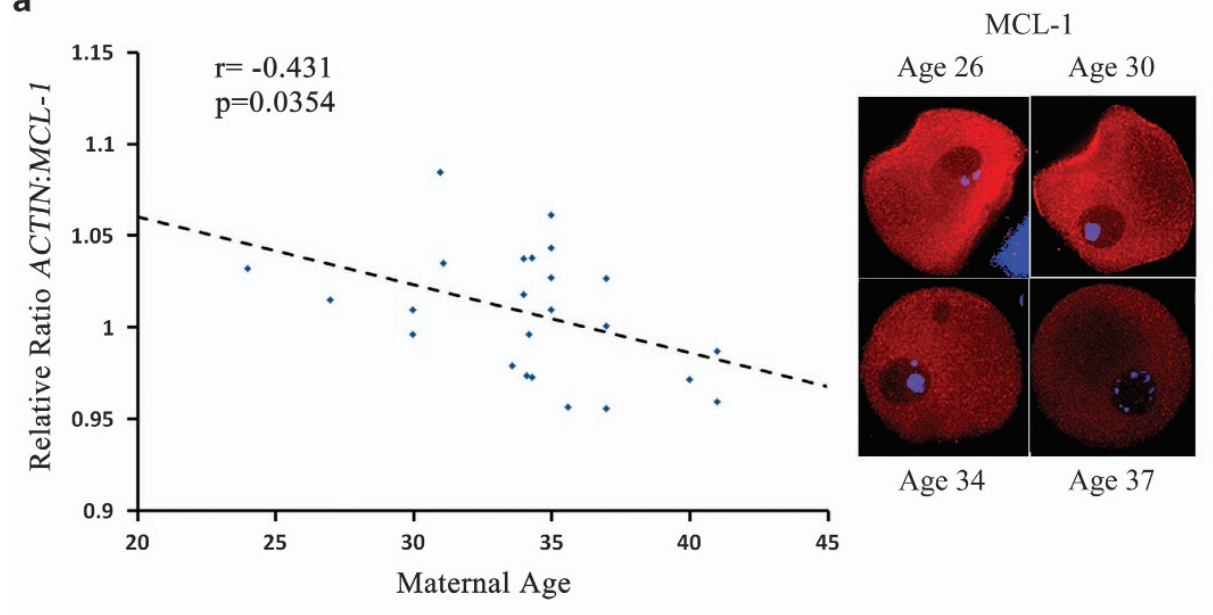

b

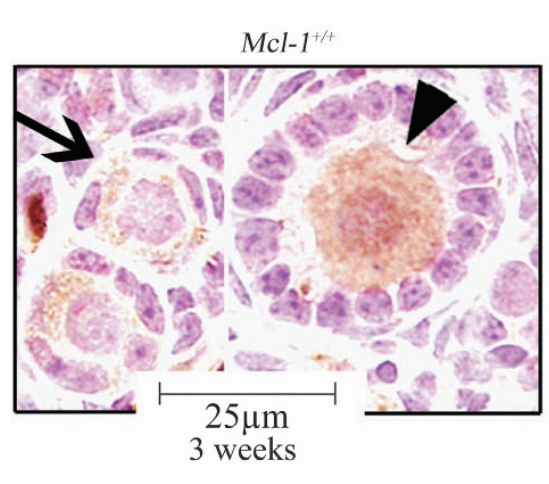

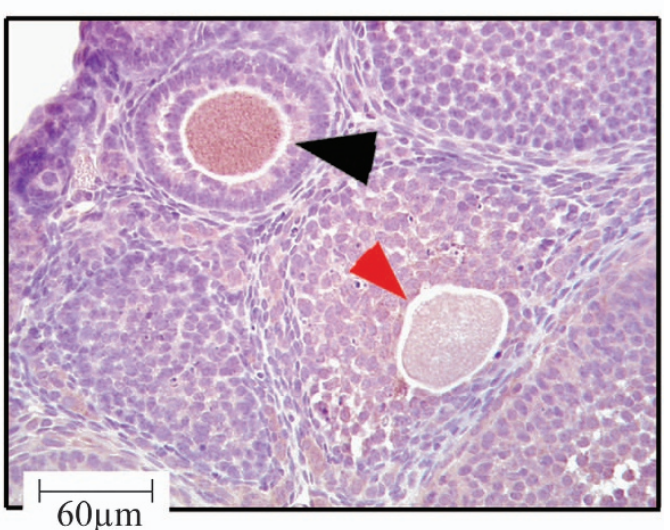

Figure 1 Patterns of MCL-1 expression in human and murine oocytes. (a) Age-associated changes in MCL-1 transcript (left) and MCL-1 protein immunoreactivity (right) in human GV oocytes from different patients. Pearson analysis revealed a negative correlation between MCL-1 transcript level and maternal age $(n=24 ; r=-0.431 ; P=0.035)$. (b) Immunohistochemical staining of MCL-1 (brown) in 3-week ovarian sections of wild-type mouse ovaries, counterstained with hematoxylin (blue). PMF oocytes (arrows), PF (PF) and PA follicles (arrowheads), and oocytes undergoing early stages of atresia (red arrowhead). At least three ovaries were stained per age group with identical expression patterns 
Oocyte-specific Mcl-1 deficiency reduces ovarian follicle pool, ovulation and fertility. To determine the functional requirement for $\mathrm{Mcl}-1$ in oogenesis, we created a mouse model with oocyte-specific excision of $\mathrm{Mcl}-1$ using zona pellucida 3 (Zp3)-Cre. We confirmed spatial and temporal activity of the Cre transgene by introduction into two reporter lines, tdTomato and lacZ/alkaline phosphatase reporter (Z/AP). Initial activation of Cre excision in oocytes was observed as early as embryonic day 17.5, with a large proportion of oocytes showing excision by postnatal day 3 (PN3; Supplementary Figure S1A). The selectivity of excision was also confirmed in the adult Z/AP reporter line, with excision in virtually all growing oocytes (Supplementary Figure S1B). We next combined the Zp3-Cre allele with floxed $\mathrm{Mcl}-1\left(\mathrm{Mcl}-1^{\mathrm{f} / \mathrm{f}}\right)$. Effective excision was confirmed by lack of MCL-1 immunoreactivity in oocytes of $\mathrm{Mcl}-1^{\mathrm{f} /-}$ : Zp3-Cre (Mcl-1 conditional knockout (Mcl-1cKO)) females (Supplementary Figure S1C).

To determine the impact of $\mathrm{Mcl}-1$ oocyte-specific deletion on fertility, $\mathrm{Mcl}-1 \mathrm{cKO}$ and control females were bred to wild-type males of proven fertility for a period of 6 months. The cumulative breeding performance of $\mathrm{Mcl}-1 \mathrm{cKO}$ dams was markedly reduced with an average of two litters during this period, and each with less than half of the pup number obtained from control females (Figure 2a, Supplementary Figure S2A). In addition, $\mathrm{Mcl}$-1cKO females did not deliver any live litters beyond 4 months of age, whereas all control females of the various genotypes were able to breed beyond 1 year of age (Supplementary Figure S2A). Mcl-1 excision was confirmed in all genotyped Mcl-1cKO offspring $(n=31)$. To establish whether the reduction in breeding performance was due to an overall reduction in the ovulatory capacity, females were primed with external gonadotropins and their ovulatory response quantitated. We used females of various age groups (at 6 and 3 months, as well as 3 weeks) as these represented mature females, at a young reproductive age and at pubertal onset, respectively. In Mcl-1cKO females, very poor ovulatory capacity was observed at 3 months and ovulation was virtually absent by 6 months (Figure 2b, Supplementary Figure S2B). Histological examination revealed a severe depletion in PMF, PF and secondary follicles (SF) and a drastic reduction in ovary size already evident at 3 months of age (Figure 2c, Supplementary Figure S2D). Furthermore, a significant reduction of PMFs and PFs was also observed in heterozygote females (Supplementary Figure S2C). Interestingly, at the onset of puberty (3 weeks), females of all genotypes ovulated comparable numbers of oocytes (Supplementary Figure S2E), yet histomorphometric analyses revealed a sharp reduction in PMFs and a significant decrease in growing follicle number (Figure $2 \mathrm{~d}$ ). The loss of growing oocytes may be partially due to an increased rate of atresia, as a significantly higher proportion of late-stage atretic follicles was observed (Figure 2f).

The loss of the resting PMF pool was the most marked outcome we have observed. At PN7, only about half of the PMFs were present with no change in the PF or SF population (Figure 2e). This, combined with terminal deoxynucleotidyl transferase dUTP nick end labeling (TUNEL) analyses of ovaries at PN1 revealing that $\mathrm{Mcl}-1$ deficiency resulted in a doubling of the number of apoptotic (TUNEL positive) oocytes compared with controls (Figure 2g, Supplementary Figure S2F), led us to conclude that $\mathrm{Mcl}-1$ is required for postnatal maintenance of the ovarian reserve.

Mcl-1 deficiency leads to elevated activation of autophagy in antral follicle population. Based on our observations that $\mathrm{Mcl}-1$ ablation resulted in mildly elevated atresia rates, and that MCL-1 expression was absent from oocytes undergoing atresia, we next assessed markers of PCD in the growing follicle pool. As PMF oocytes of $\mathrm{Mcl}-1 \mathrm{cKO}$ ovaries die via an apparent apoptotic cell death, we set to investigate whether antral GV oocytes also activate this death pathway. Relative to controls, Mcl-1cKO oocytes exhibited an increase in $\mathrm{Bcl}-2$-associated $X$ protein (BAX)-NT, indicative of a conformational change in the $\mathrm{N}$-terminus of $\mathrm{BAX}$ and potentiating oligomerization; however, downstream hallmarks of mitochondrial engagement (e.g., cytochrome $\mathrm{C}$ or apoptosis-inducing factor (AIF) release) showed no significant change, with no increase in pan-caspase activity (Supplementary Figures S3A-D). This data effectively demonstrates that despite $\mathrm{BAX}$ activation, there is no subsequent instigation of the apoptotic cascade in antral Mcl-1cKO GV oocytes.

As follicle atresia exhibits hallmarks of both autophagic (PCD type 2) and apoptotic (PCD type 1) cell death ${ }^{13}$ and Mcl-1 has been previously linked to inhibition of both pathways, ${ }^{26-28}$ we turned to the assessment of markers of autophagic activation in GV oocytes. Induction or phosphorylation of Beclin-1/BECN-1 has been shown to initiate autophagosome formation, ${ }^{29}$ and MCL-1 binding and inhibition of Beclin-1 prevented autophagy. ${ }^{27} \mathrm{Mcl}-1 \mathrm{CKO}$ oocytes displayed an increase in size and number of Beclin-1 foci, indicating an increase in Beclin-1-associated vesicle formation (Figure 3a,Supplementary Figure S3F). In addition, microtubule-associated protein 1 light chain (MAP1LC3A), involved in autophagosome membrane elongation, ${ }^{30}$ was elevated in $\mathrm{Mcl}-1 \mathrm{cKO}$ GV oocytes (Supplementary Figure $\mathrm{S} 3 \mathrm{E})$. We next examined markers of late autolysosomes. Lysosome-associated membrane proteins 1 and 2 (LAMP-1, LAMP-2) are an essential part of autophagosome maturation, autolysosome formation and lysosomal fusion. ${ }^{31} \mathrm{Mcl}-1 \mathrm{cKO}$ GV oocytes showed an increased number of foci positive for both Beclin-1 and LAMP-2 (Figure 3a). Colocalization analyses performed on $3 \mathrm{D}$ rendered oocyte sections confirmed a significant increase in Beclin-1/LAMP-2 colocalization in $\mathrm{Mcl}-1 \mathrm{cKO} \mathrm{GV}$ oocytes. These colocalization analyses also demonstrated that oocytes possessing just one $\mathrm{Mcl}-1$ allele $\left(\mathrm{Mcl}-1^{f /+}\right.$ : Zp3-Cre), display an intermediary phenotype (Supplementary Figure S3F). The amplified abundance of autolysosome structures in $\mathrm{Mcl}-1 \mathrm{cKO}$ oocytes was further validated by transmission electron microscopy (Figure $3 b$ ). The elevation in number of Beclin-1-positive foci, LC-3 immunoreactivity and Beclin-1/LAMP-2 colocalized structures, indicates an augmentation of autophagosome and autolysosome formation, triggered by $\mathrm{Mcl}-1$ deficiency.

Ovulated Mcl-1-deficient oocytes display mitochondrial dysfunction and chromosomal abnormalities. Despite the marked loss of the ovarian pool at a young age, $\mathrm{Mcl}-1 \mathrm{cKO}$ females do ovulate and breed, albeit at reduced 
efficiencies. As oocyte quality and reproductive competence have been suggested to rely on effective mitochondrial metabolism, ${ }^{32-34}$ and $\mathrm{Mcl}-1$ has been implicated in regulation of mitochondrial function in somatic cells, ${ }^{28,35,36}$ we assessed these parameters in $\mathrm{Mcl}-1 \mathrm{cKO}$ ovulated oocytes. Using a mitochondrial membrane potential sensitive dye, we observed two distinct patterns of DePsipher fluorescence (Figure 4a). Control oocytes displayed a strong centrally localized inactive mitochondrial pool (green) with enrichment of polarized mitochondria (red) in the cortical region (pattern 1). a

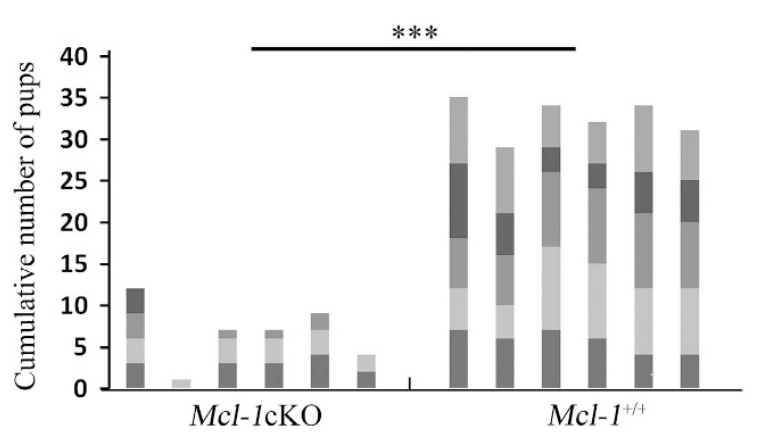

C

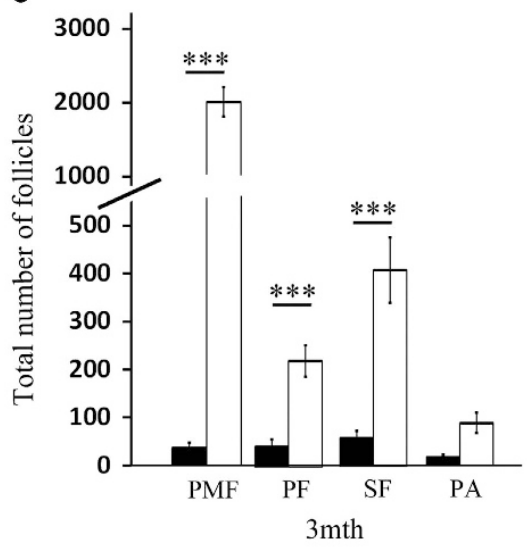

d

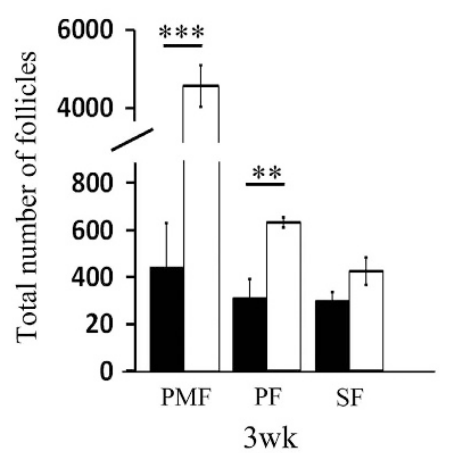

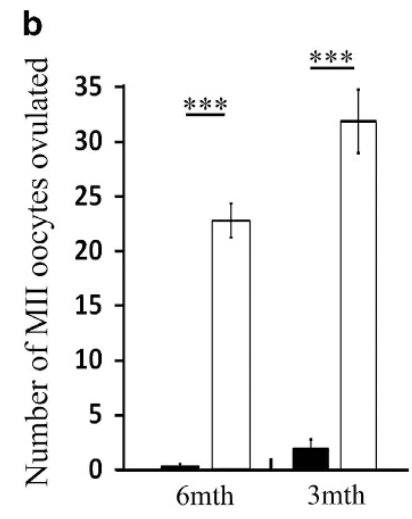

e f

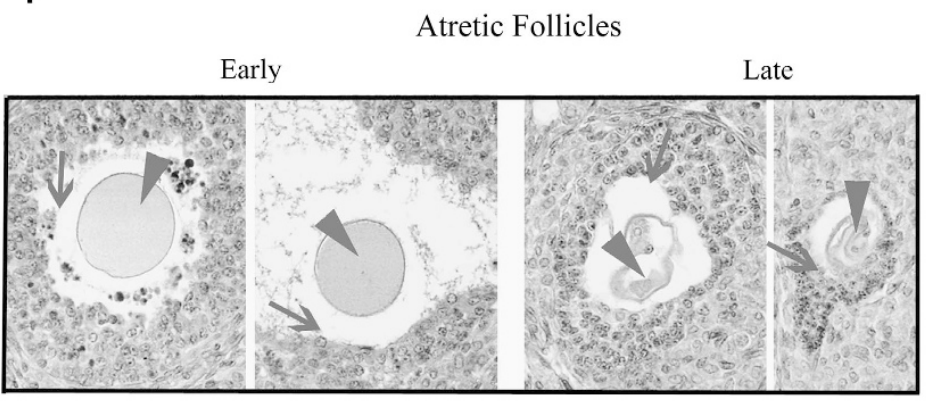

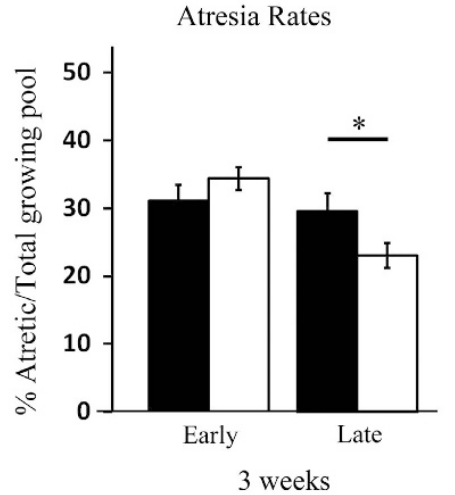

g

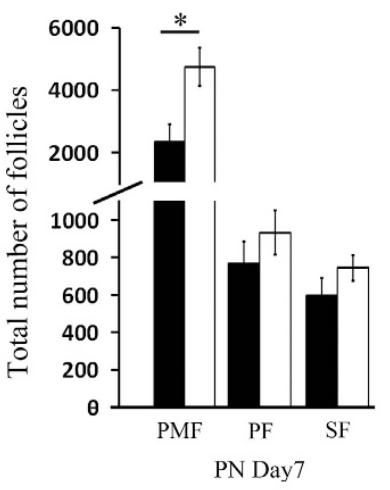

Atresia Rates

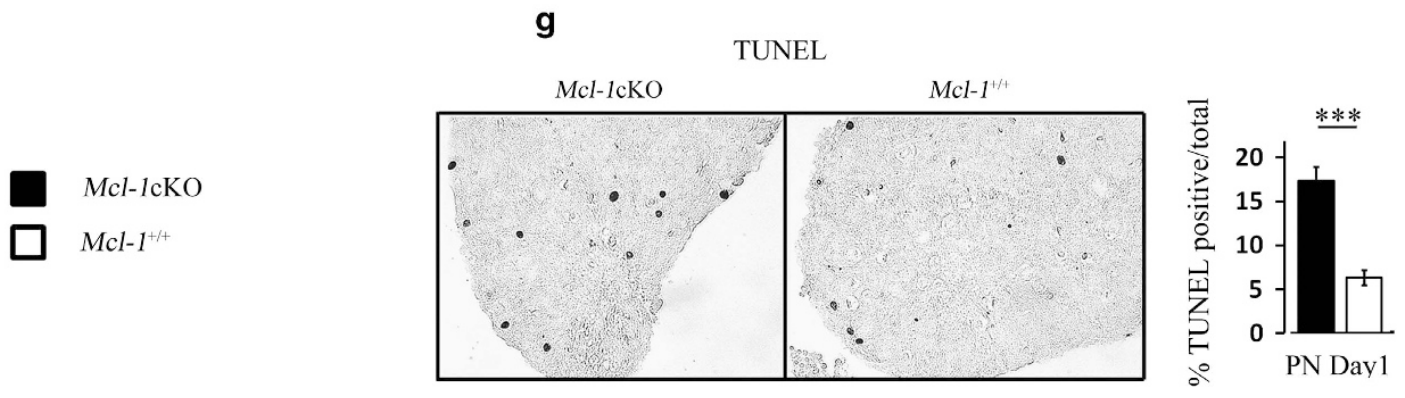


Conversely, many $\mathrm{Mcl}$-1cKO oocytes exhibited a weak diffuse distribution of inactive mitochondria (green) and extremely few peripherally active polarized mitochondria (red) (pattern 2). Moreover, the number of peripheral polarized mitochondrial foci was significantly decreased in Mcl-1cKO oocytes compared with controls (Figure 4a, Supplementary Figure S4A). A decline in the respiring mitochondrial pool, evidenced by decreased MitoTracker Red intensity was also observed in $\mathrm{Mcl}$-1cKO metaphase II (MII) oocytes (Figure 4b,Supplementary Figure S4C). This decrease was significant despite a marked elevation in the total mitochondrial pool demonstrated by increased
MitoTracker Green (Supplementary Figure S4B), a dye that binds mitochondrial membranes irrespective of their activity. In measuring mitochondrial output, Mcl-1cKO MII oocytes presented with a sharp reduction in levels of the TCA cycle substrates fumarate and malate (Figure 4c), but showed no apparent difference in total ATP level or citrate (Supplementary Figure S4D), when compared with control, implying no change in available energy. In addition, total reactive oxygen species (ROS) and mitochondrialderived superoxides were markedly increased (Figure 4d, Supplementary Figures S4E and 4F). High ROS levels can be indicative of defective antioxidant machinery or an inhibition/ a
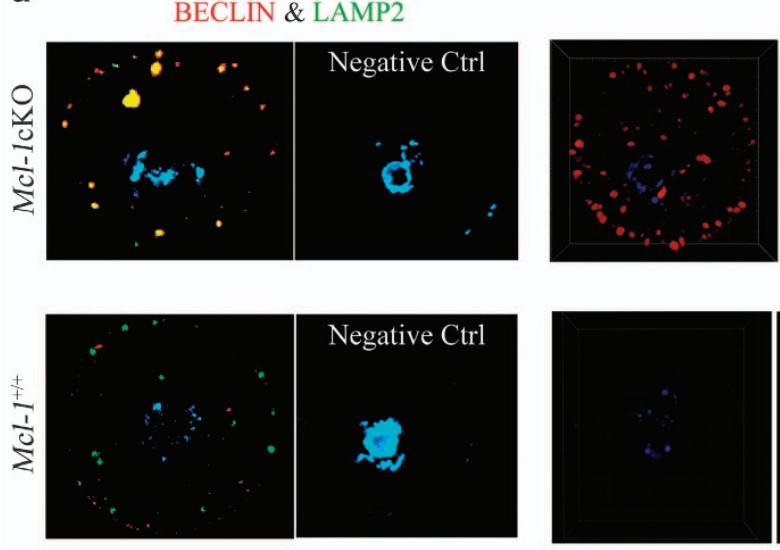

3D Rendering of BECLIN and LAMP2
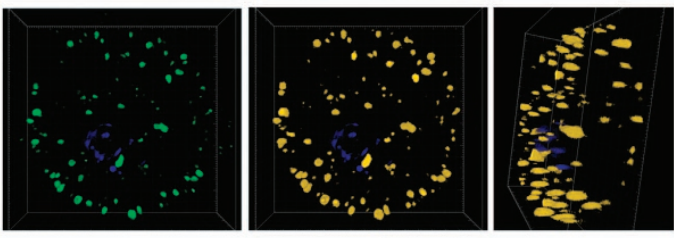

Colocalized Pixels

b

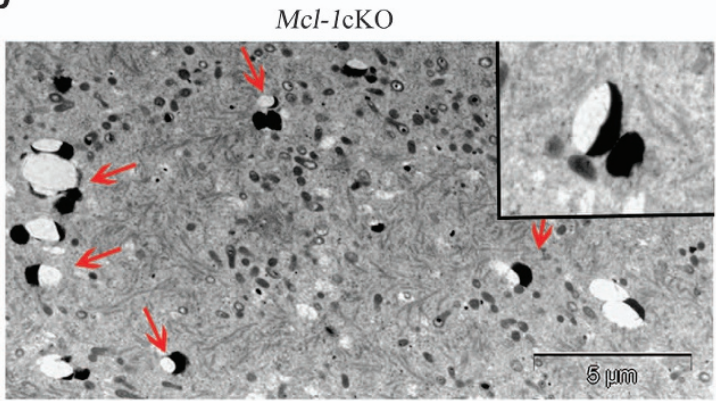

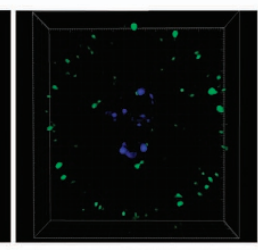
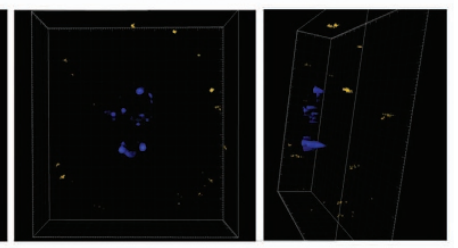

$\mathrm{Mcl}-1^{+/+}$

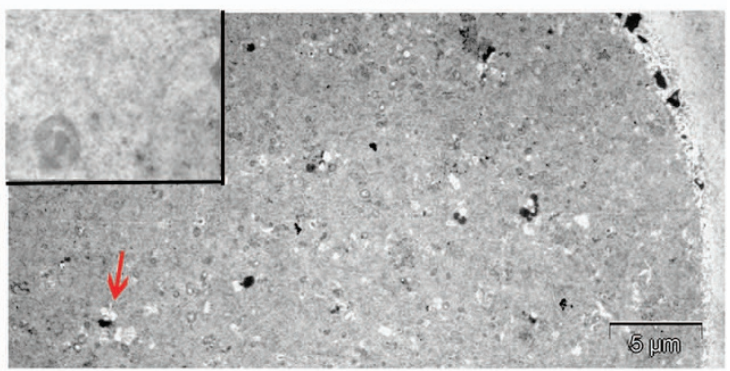

Figure 3 Markers of autophagy in GV oocytes. (a) Confocal image of Beclin-1 (red) and LAMP-2 (green) foci in isolated GV oocytes from 3-week ovaries, counterstained with $\mathrm{DAPI}$ (blue). To determine colocalization, 3D rendering of all layers was performed on $n=19 \mathrm{Mcl}-1 \mathrm{cKO}$ and $n=13 \mathrm{Mcl}-1^{+/+} \mathrm{GV}$ oocytes with colocalized (yellow) pixels displayed. Coefficients of colocalization for Mcl-1cKO oocytes compared with $M c l-1^{+/+}$(Pearsons (PCC) $=0.51 \pm 0.05$ to $0.17 \pm 0.07$, respectively; $P<0.001$ ) (Manders A (MCCA) $=0.098 \pm 0.017$ to $0.058 \pm 0.01$, respectively; $P<0.05$ ) (Manders B (MCC-B) $=0.079 \pm 0.014$ to $0.032 \pm 0.005$, respectively; $P<0.05)(b)$ Transmission electron microscopy (TEM) of Mcl-1cKO and wild-type control GV oocytes with lysosome-associated vesicles (red arrows) indicated

Figure 2 Breeding performance, ovulation rates and histomorphometric analyses of ovaries. (a) Cumulative pup number from $M c l-1 \mathrm{cKO}(n=6)$ and $M c l-1^{+/+}(n=6)$ females bred with wild-type males at 4 weeks for 6 -month trial. Columns represent individual females with each varied color segment indicative of individual litters. (b) Ovulation rates from primed 6-month $\mathrm{Mcl}-1 \mathrm{CKO}(n=12)$, compared with $\mathrm{Mcl}^{+1^{+/+}}(n=18)$ control females and 3-month $\mathrm{Mcl}-1 \mathrm{CKO}(n=20)$, compared with $M c l-1^{+/+}(n=12)$ females. Values represent average oocytes ovulated per female \pm S.E.M. (c) Histomorphometric analyses of PMF, PF, SF and PA follicles in 3-month $M c l-1 c K O(n=5)$, compared with $M c l-1^{+/+}$ $(n=3)$ females; (d) in 3-week ovaries of Mcl-1cKO $(n=5)$ to ${\mathrm{Mcl}-1^{+/+}}_{(n=4)}$ females; $(\mathbf{e})$ and in day 7 (PN7) Mcl-1cKO $(n=4)$, compared with Mcl-1 ${ }^{+/+}(n=4)$ females. Values in $\mathbf{c}, \mathbf{d}$ and $\mathbf{e}$ represent average number of follicles/ovary \pm S.E.M. (f) Representative stages of follicle atresia (left) in ovarian sections stained with methyl green, display granulosa cell death/detachment (arrows) preceding oocyte shrinkage (arrowhead). Both early and late-stage follicle atresia rates of $M c l-1 \mathrm{cKO}(n=4)$, compared with $M c l-1^{+/+}(n=5)$ ovaries. Atretic follicles were taken as a proportion of the total post-secondary growing follicles and separated into advanced stage (late) or early/moderate stage follicle atresia. (g) Death rates based on TUNEL stain of PN1 ovaries of Mcl-1cKO $(n=4)$ compared with $M c l-1^{+/+}(n=3)$ females counterstained with methyl green. Values represent average percentage of TUNEL positive/total PMF per section \pm S.E.M. Significance denoted by number of stars $\left({ }^{\star} P<0.05,{ }^{* \star} P<0.01,{ }^{* * \star} P<0.001\right)$. Genotypes in legend apply to all graphs 
block in the electron transport chain, ${ }^{37,38}$ and elevated ROS and impaired oxidative phosphorylation have been linked with defective development and meiotic spindle defects. ${ }^{33,39-41}$ Microtubule staining of the meiotic spindle apparatus revealed increased chromosomal misalignments in $\mathrm{Mcl}-1 \mathrm{cKO}$ oocytes compared with controls with misaligned chromosome (s) not attached to spindle (Figure 4e, Supplementary Figure S4G). a

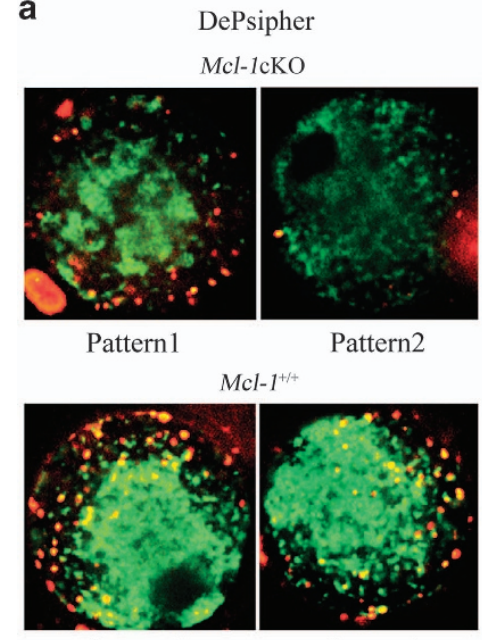

Pattern1

Pattern1

b

MitoTracker Red
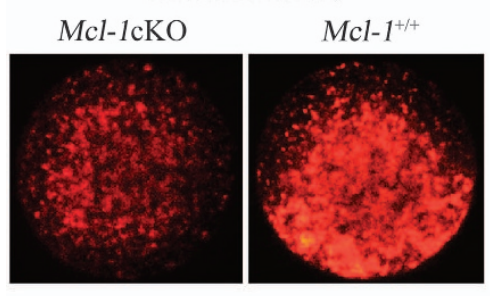

d

ROS

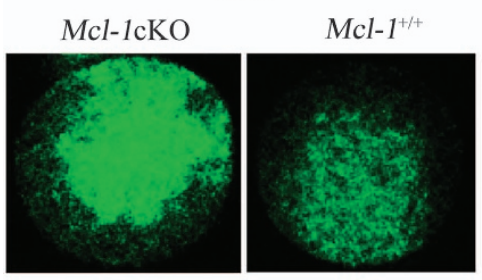

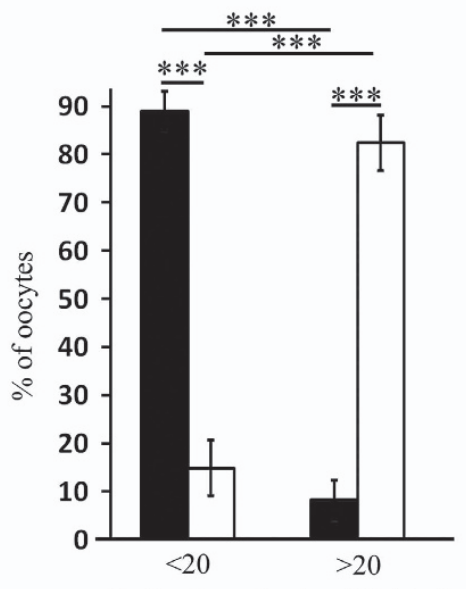

\# of active mitochondrial foci

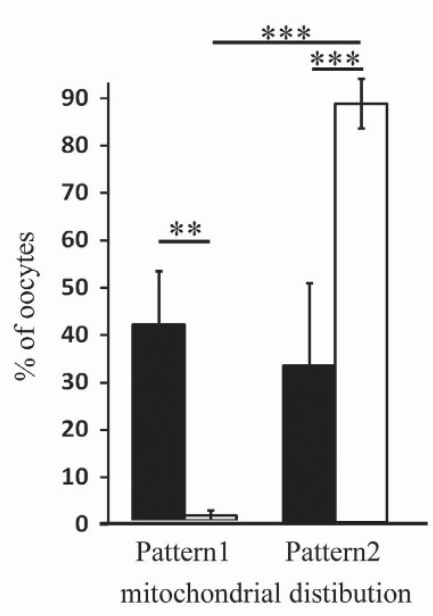

C
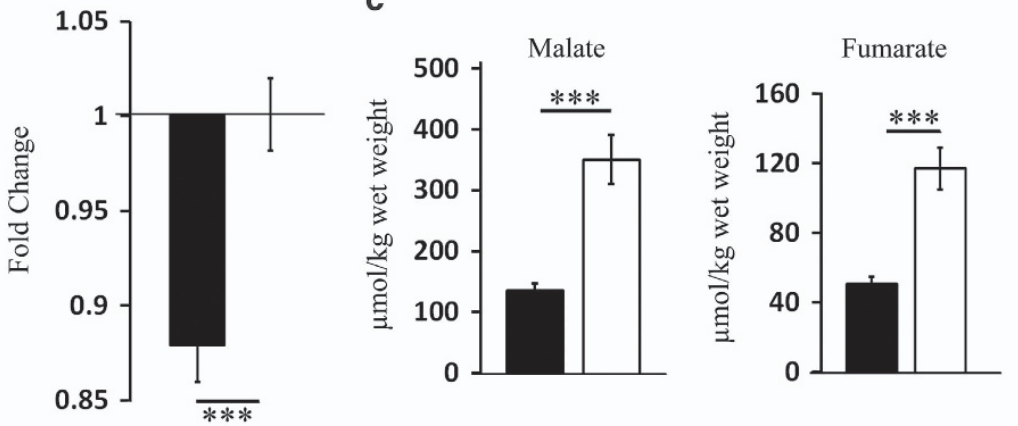
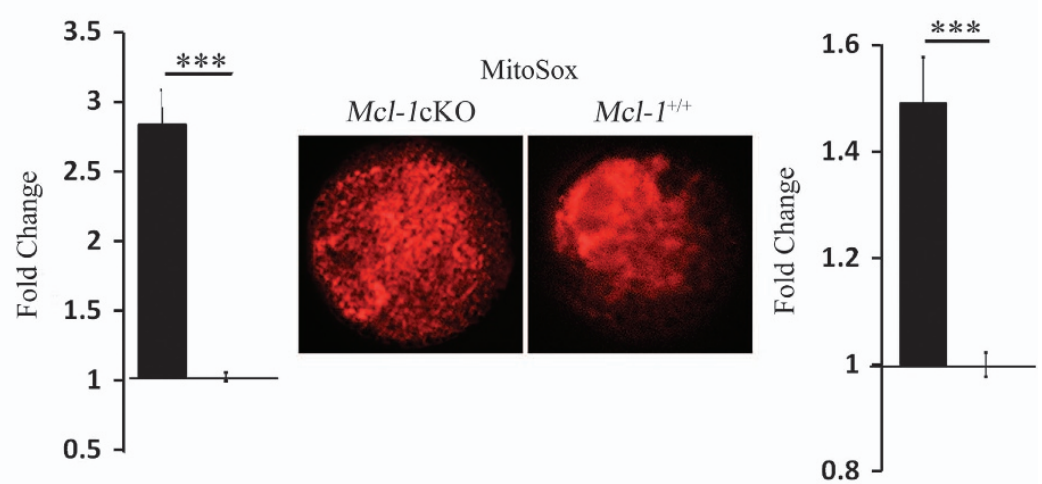

e

Chromosomal Misalignment
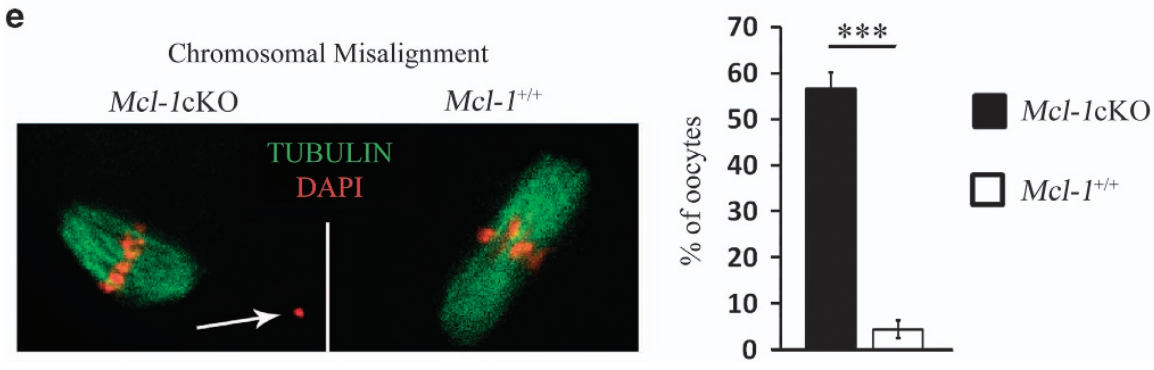
Mcl-1-deficient ovulated oocyte pool displays elevated markers of apoptosis and increased fragmentation rates. Defective mitochondrial bioenergetics are expected to impair oocyte survival, particularly after ovulation, when cumulus cells stop directly providing oocytes with nutrients. ${ }^{42}$ Thus, we decided to investigate whether the viability of ovulated oocytes was compromised because of $\mathrm{Mcl}-1$ deficiency. In vitro culture revealed an inability of $\mathrm{Mcl}-1 \mathrm{cKO} \mathrm{MII}$ oocytes to sustain meiotic arrest over $24 \mathrm{~h}$ and an increased propensity to undergo cellular fragmentation (Figure 5a). As cellular fragmentation of oocytes is thought to occur via apoptosis, ${ }^{43}$ we explored whether hallmarks of activation of the apoptotic pathway could be detected. Indeed, ovulated oocytes displayed an increase in BAX-NT (Figure 5b, Supplementary Figure S5A) with no change in total BAX levels (Supplementary Figure S5A), accompanied by an increase in mitochondrial cytochrome $C$ release (Figure $5 \mathrm{c}$, Supplementary Figure S5B) and caspase activity (Figure 5d, a

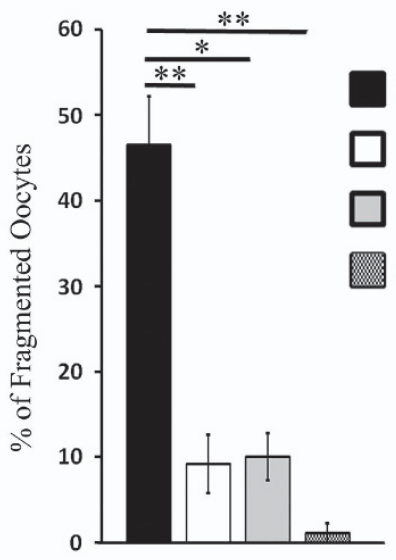

C

Cytochrome C Release

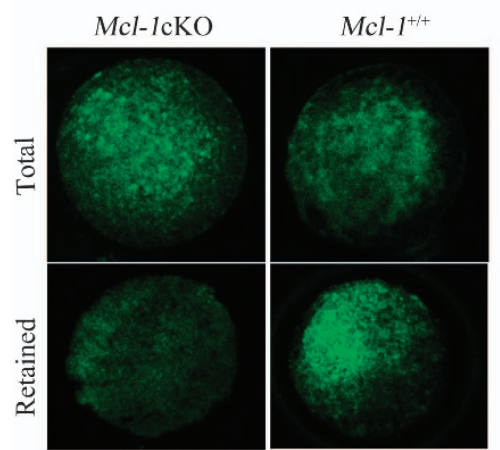

Mcl-1cKO

$\mathrm{Mcl}-1^{+/++}$

$\mathrm{Mcl}-1^{+/+}: \mathrm{Zp} 3-\mathrm{Cre}$

$\mathrm{Mcl}-1^{\mathrm{fl+}}: \mathrm{Zp} 3-\mathrm{Crc}$

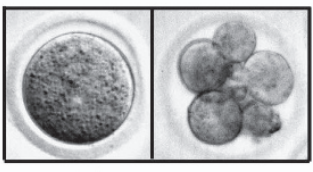

Healthy Fragmented

b

\section{Active BAX}
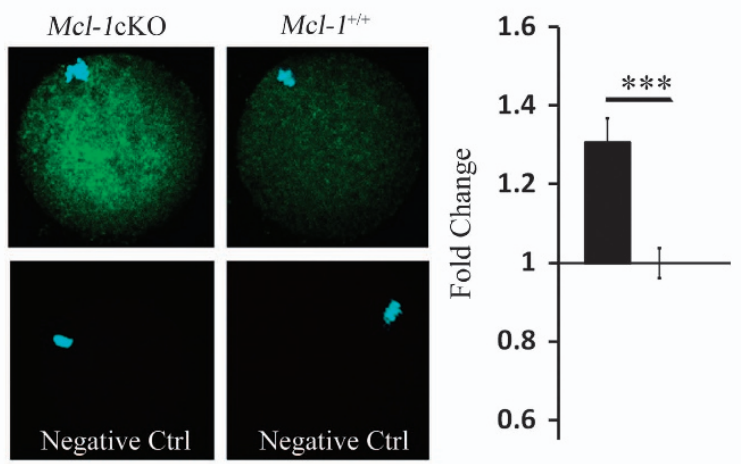

d

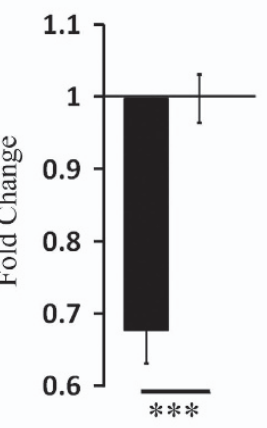

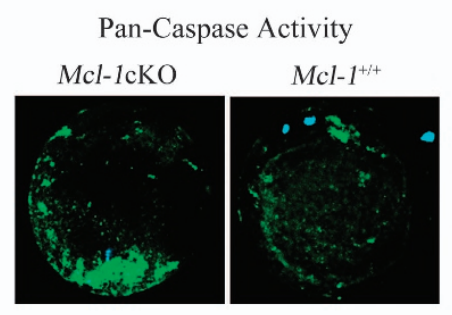

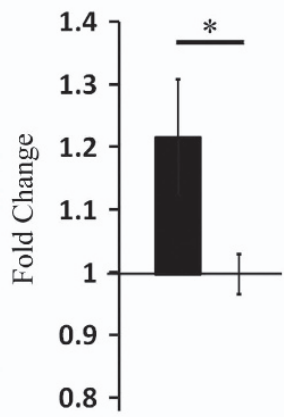

Figure 5 Markers of apoptosis in ovulated oocytes. (a) Oocytes obtained from Mcl-1cKO $(n=6), M c l-1^{+/+}(n=9), M c l-1^{+/+}: \mathrm{Zp3}-\mathrm{Cre}(n=8)$, and Mcl- $1^{7 /+}: \mathrm{Zp3}-\mathrm{Cre}(n=4)$ females ( $n=104, n=180, n=241$ and $n=69$ oocytes, respectively) were scored $24 \mathrm{~h}$ after in vitro culture for percentage of fragmented oocytes \pm S.E.M. per female/genotype. (b) Mcl-1cKO $(n=12)$ and ${\mathrm{Mcl}-1^{+/+}}_{(n=13)}$ MIl oocytes were stained with anti-BAX-NT (green), counterstained with DAPI (blue). Mean intensity was quantitated as fold change of RFUs per oocyte \pm S.E.M. normalized to average $M c l-1^{+/+}$value. (c) $M c l-1 \mathrm{cKO}(n=19)$ and $M c l-1^{+/+}(n=33)$ Mll oocytes were stained for total, and $M c l-1 \mathrm{cKO}(n=13)$ and $M c l-1^{+/+}$ $(n=31)$ oocytes, were stained for retained Cytochrome c (green). Values represent the average fold change of RFUs per oocyte \pm S.E.M. of retained/total cytochrome c, normalized to average ${\mathrm{Mcl}-1^{+/+}}$value. (d) $\mathrm{Mcl}-1 \mathrm{cKO}(n=16)$ and $\mathrm{Mcl}-1^{+/+}(n=13)$ Mll oocytes were stained for pan-caspase activity (green). Values indicate average fold change of RFUs per oocyte \pm S.E.M. normalized to average $M c l-1^{+/+}$value $\left({ }^{*} P<0.05,{ }^{* \star} P<0.01,{ }^{* * \star} P<0.001\right)$. Genotypes in legend apply to all graphs

Figure 4 Markers of mitochondrial function and chromosomal alignment. (a) $M c l-1 \mathrm{CKO}(n=57)$ and $\mathrm{Mcl}-1^{+/+}(n=85)$ MIl oocytes were stained with a potential dependent mitochondrial dye (DePsipher). Values in graph (left) represent a proportion (\%) of oocytes with few $(<20)$ or numerous $(>20)$ polarized (red) mitochondrial foci \pm S.E.M. per $\mathrm{Mcl}-1 \mathrm{CKO}$ or $\mathrm{Mcl}_{-1++}$ oocytes. Values in graph (right) represent the proportion (\%) of oocytes with mitochondrial distribution separated into pattern 1 or pattern $2 \pm$ S.E.M. (b) Mean intensity of $\mathrm{Mcl}-1 \mathrm{CKO}(n=68)$ and ${\mathrm{Mcl}-1^{++}}_{(}(n=52)$ MIl oocytes stained with MitoTracker Red. Values represent average fold change \pm S.E.M. of relative fluorescence units (RFUs) per oocyte, normalized to wild type. (c) Mcl-1cKO $(n=15)$ and $M c l-1^{+/+}(n=15)$ oocytes were assayed for levels of TCA cycle substrates malate and fumarate. Values represent average metabolite level $(\mu \mathrm{mol})$ per oocyte wet weight $(\mathrm{kg}) \pm$ S.E.M. (d) MIl oocytes were stained with DCFDA (green) $\left(\mathrm{Mcl}-1 \mathrm{cKO}(n=20)\right.$ and $M \mathrm{Ml}-1^{+/+}$ $(n=17))$ or with MitoSox (red) (Mcl-1cKO $(n=20)$ and $\left.\mathrm{Mcl}^{+1^{+/+}}(n=24)\right)$. Values represent average fold change \pm S.E.M. of RFUs per oocyte, normalized to wild type. (e) MII oocytes were stained with DAPI (red) and anti-tubulin (green) to visualize chromatin and spindle; chromosomal misalignments (white arrow) from $M c l-1 \mathrm{cKO}(n=84)$ and $\mathrm{Mcl}-1^{+/+}$ $(n=89)$ oocytes were quantitated. Values represent percentage of oocytes with misaligned chromosomes/total oocyte pool. $\left({ }^{\star} P<0.05,{ }^{* *} P<0.01,{ }^{* \star *} P<0.001\right)$. Genotypes in legend apply to all graphs 
a

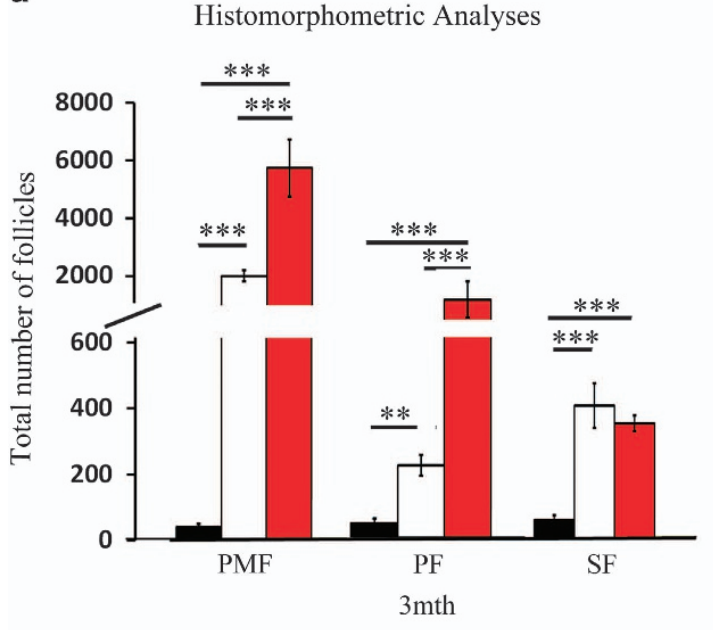

C

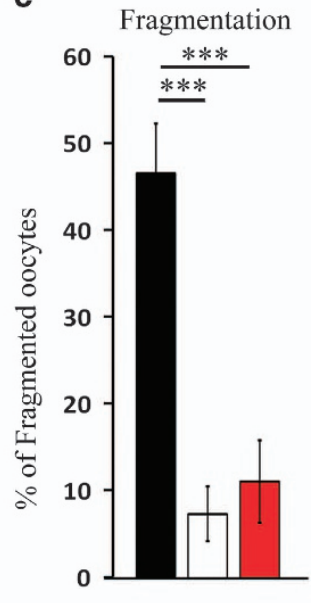

d

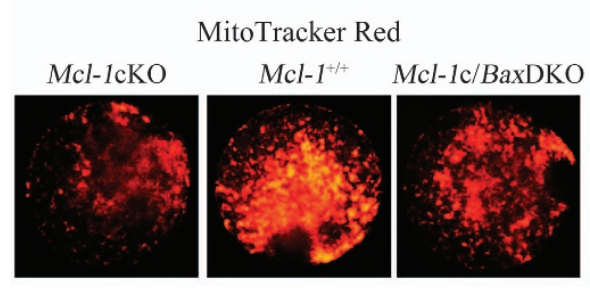

Mcl-1c/BaxDKO

${\mathrm{Mcl}-\mathrm{I}^{+/+}}^{-1}$

b

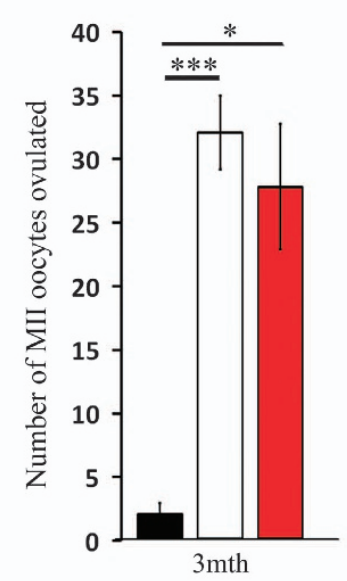

Ovulation Rates
Mcl- $1 \mathrm{cKO}$

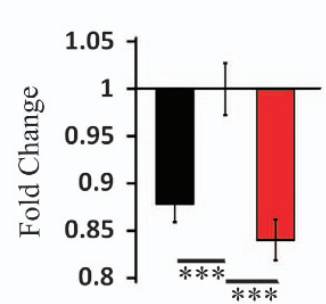

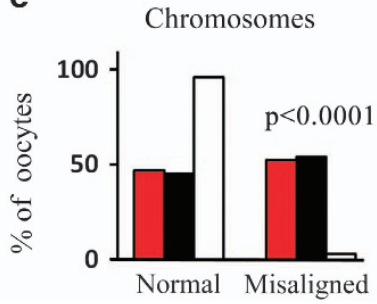

Figure 6 Rescue of $M C l-1$-deficient follicle loss by concurrent Bax ablation. (a) Histomorphometric analyses of 3-month ovaries in $M c l-1 \mathrm{cKO}(n=5), M c l-1^{+/+}(n=3)$ and $M c l-$ $1 \mathrm{c} / \mathrm{BaxDKO}(n=3)$ females. Values represent average follicle number in ovary per genotype \pm S.E.M. (b) Ovulation rates of $M c l-1 \mathrm{cKO}(n=20), M c l-1^{+/+}(n=12)$ and $M c l-1 \mathrm{cl}$ $\operatorname{BaxDKO}(n=3) 3$-month females. Values represent average number of ovulated oocytes \pm S.E.M. (c) Mll oocytes from Mcl-1cKO $(n=6), M c l-1^{+/+}(n=10)$ and $M c l-1 c / B a x D K O$ $(n=3)$ females $(n=104, n=180, n=48$ oocytes, respectively) cultured for $24 \mathrm{~h}$ and values represent the average percentage of fragmented oocytes per female \pm S.E.M. (d) Mcl-1cKO $(n=50), \mathrm{Mcl}-1^{+/+}(n=33)$, and Mcl-1c/BaxDKO $(n=49)$ MII oocytes were stained with MitoTracker Red. Values represent fold change of average RFUs per oocyte \pm S.E.M. normalized to average $M \mathrm{MCl}^{+1^{+/+}}$value. (e) Chromosomal misalignments in $\mathrm{Mcl}-1 \mathrm{cKO}(n=70), \mathrm{Mcl}^{+1^{+/+}}(n=89)$ and $\mathrm{Mcl}-1 \mathrm{c} / \mathrm{BaxDKO}(n=19)$ 0ocytes were quantitated (from $n=4, n=5$ and $n=2$ animals, respectively). Values represent percentage of oocytes with misaligned or normal chromosomes/total oocyte pool. Mcl-1c/ BaxDKO data were all graphed against $\mathrm{MCl}-1 \mathrm{cKO}$ and $\mathrm{Mcl}-1^{++}$data from Figures 2,4 and $5 .\left({ }^{\star} P<0.05,{ }^{\star \star} P<0.01,{ }^{* \star} P<0.001\right)$. Genotypes in legend apply to all graphs

Supplementary Figure S5C). Thus, Mcl-1 deficiency in ovulated MII oocytes leads to an activation of the apoptotic cascade, and an increased proclivity for these compromised oocytes to fragment.

Concurrent deletion of Bax rescues ovarian follicle loss and increased fragmentation rate but does not impact mitochondrial dysfunction or chromosome abnormalities. Deletion of the pro-apoptotic $\mathrm{Bcl}-2$ family member $\mathrm{Bax}$ rescues the primordial germ cell loss induced by ablation of the anti-apoptotic $\mathrm{BCl}-\mathrm{x}^{20}$ By itself, Bax deficiency has been linked to increased primordial germ cell survival, ${ }^{44}$ resulting in an increase in follicular endowment ${ }^{45}$ and extended ovarian function to advanced chronological age. ${ }^{46}$ In order to evaluate whether Bax deficiency would also rescue loss of the ovarian reserve in $\mathrm{Mcl}-1 \mathrm{cKO}$ females, we crossed a Baxdeficient line to our $\mathrm{Mcl}-1 \mathrm{cKO}$ mouse line. Ovaries from 3 month $\mathrm{Bax}^{-/-}$(Bax knockout (BaxKO)), $\mathrm{Mcl}_{-1} \mathbf{1}^{t /-}$ : $\mathrm{Bax}^{-/-}$: Zp3-Cre (double knockout with conditional Mcl-1 and total Bax deletion (Mcl-1c/BaxDKO)), Mcl-1cKO females and controls were used for histomorphometric ovarian analyses. Bax deficiency alone substantially increased the cohort of
PMFs; and the Mcl-1c/BaxDKO ovarian reserve resembled BaxKO ovaries in all follicle numbers, implying a rescue of the Mcl-1-deficient oocyte phenotype (Figure 6a, Supplementary Figure S6A). Moreover, $\mathrm{Mcl}$-1c/BaxDKO females displayed restored ovulation rates (Figure 6b, Supplementary Figure S6B), and a resiliency of MII oocytes against cellular fragmentation during in vitro culture, akin to wild-type controls and BaxKO (Figure 6c, Supplementary Figure S6C). However, despite a very robust rescue to the ovarian follicle pool, Bax deficiency was not able to restore mitochondrial functionality (Figure 6d, Supplementary Figure S6D) or correct the choromosomal misalignments (Figure 6e) in ovulated oocytes, indicating that, in oocytes, $\mathrm{Mcl}-1$ regulates additional aspects of cellular and mitochondrial physiology independent of Bax or cell death. Indeed, breeding trials of older (4- to 8-month old) BaxKO or Mcl-1c/BaxDKO mice revealed a longer breeding expectancy, but not an increase in the number of pups, as litter size remained $<2$. Although no $\mathrm{Mcl}-1 \mathrm{CKO}$ mice $(n=8)$ produced litters after 4 months of age, two out of three $\mathrm{Mcl}-1 \mathrm{c} / \mathrm{BaxDKO}$ females produced at least one litter during the 3-month breeding trial (Supplementary Table S1). 


\section{Discussion}

Limited ovarian phenotypes caused by deletion of antiapoptotic $\mathrm{Bcl}-2$ members ${ }^{16-19,21}$ has led to the postulation that they are either uninvolved or redundant in postnatal oocyte survival. $\mathrm{Mcl}-1$ has a well-documented cell survival role for numerous somatic cell types; ${ }^{36,47,48}$ however, its necessity for long-term germ cell viability has not been established. We present evidence of $\mathrm{Mcl}-1$ as the first pro-survival $\mathrm{Bcl}-2$ member required for life-time maintenance of the ovarian reserve and stage-specific inhibition of varying modes of oocyte cell death.

As demonstrated above, cytoplasmic MCL-1 expression increases during the transition from PMF to PF, and continues to accumulate with sustained follicle growth; but oocytes in atretic follicles lack MCL-1 indicating that downregulation of MCL-1 could precede initiation of oocyte atresia. Mcl-1 appears to mediate PMF survival by antagonizing Bax action, as Mcl-1 deficiency-induced PMF loss by apoptotic oocyte death, was restored by concurrent Bax ablation. Those Mcl-1deficient oocytes that escape the early PMF demise and begin to grow, exhibit increased markers of cellular autophagy without apoptotic activation, resulting in only mildly elevated rates of atresia. This lack of apoptotic activation may in part be due to additional anti-apoptotic Bcl-2 members neutralizing activated $B A X$ in the absence of $M C L-1$, or via additional undetermined mechanisms. We also show that the ability to initiate the apoptotic cascade does not occur till ovulation, as meiotic completion is essential for apoptotic activation. ${ }^{49,50}$

We postulate that $\mathrm{Mcl}$-1-deficient GV oocytes activate the autophagic machinery in response to mitochondrial dysfunction accompanied by disruption of metabolic machinery, however, we cannot exclude the possibility that Mcl-1 can directly regulate autophagy. Deletion of $\mathrm{Mcl}-1$ has been recently linked to elevated mitochondrial dysfunction and activation of either autophagy or apoptosis in a stress- and cell-specific manner in cardiomyocytes, cortical neurons and MEFs. ${ }^{28,35,36}$ When evaluated, these phenotypes were accompanied by disruption of mitochondrial membrane potential, defective ATP output and production of superoxides. Mitochondrial bioenergetic output and functionality have long been considered essential factors in mediating oocyte quality and reproductive competence. ${ }^{32,33} \mathrm{Mcl}-1 \mathrm{cKO}$ oocytes display enhanced mitochondrial dysfunction; with elevated superoxides, impaired mitochondrial activity and reduced fumarate and malate, associated with chromosome abnormalities and elevated MII oocyte fragmentation; the last phenotype alone being mitigated by concurrent Bax deficiency. These mitochondrial phenotypes may be regulated by a newly identified matrix-localized isoform of MCL-1, associated with prevention of defective complex II enzymatic activity and disrupted supercomplex assembly. ${ }^{35}$ Confirmation of a role for this isoform in oocytes is yet to be determined. As oocyte-cumulus cell contact is required for the continuance of meiotic arrest, oocyte growth ${ }^{51,52}$ and regulation of metabolite supply to the oocyte, ${ }^{53-56}$ it is conceivable that maintained granulosa/ cumulus cell support permits the Mcl-1-deficient immature oocytes to prolong their demise and, in conjunction with activation of autophagy, compensates for associated metabolic deficiencies. Subtle changes in ATP levels ${ }^{57,58}$ and relief of MCL-1 inhibition of Beclin, ${ }^{27,28}$ could be sufficient for autophagic activation in $\mathrm{Mcl}$-1-deficient oocytes.

Additional studies are required to elucidate whether the maintenance of mitochondrial dysfunction with concomitant Bax deficiency may be due to a BAX-independent function of MCL-1, an additional role of BAX in mitochondrial function ${ }^{59}$ or some combination of both. As breeding was prolonged but not fully rescued by Bax deficiency, it indicates that although oocyte death could be prevented by Bax ablation, additional roles of $\mathrm{Mcl}-1$, independent of Bax, are present, and may also govern embryo development. ${ }^{60}$ It would also be very informative to determine if depletion of Bok, another multichannel pro-apoptotic member of Bcl-2 gene family, could rescue the developmental competence of $\mathrm{Mcl}$-1-deficient oocytes. It should also be noted that in keeping with previous data, ${ }^{46}$ Bax-deficient females do display variable breeding performance.

Fertility and reproductive proficiency has been well established to rely on the maintenance of the ovarian reserve in addition to preservation of oocyte quality. We observed a correlation between maternal age and $M C L-1$ expression levels in human oocytes, and our animal model demonstrates that oocyte-specific ablation of $\mathrm{Mcl}-1$ results in the accumulation of defects associated with compromised oocyte quality. ${ }^{6,7,9,10,61}$ Furthermore, we establish that $\mathrm{Mcl}-1$ has the defining role in mediation of oocyte survival via protection of the postnatal PMF pool, in addition to the growing and ovulated oocyte pool. Thus, investigation into modulation of Mcl-1 expression by oocytes could prove informative in understanding what factors may contribute to POF and agerelated oocyte loss.

\section{Materials and Methods}

Animals. Mcl- $1^{\text {tm } 3 S \text { jk }}\left(\mathrm{MCl}^{-1^{t}}\right)^{47}$ mice carrying the floxed allele were obtained from the breeding colony of Dr. Korsmeyer and were intercrossed to mice carrying the $\mathrm{Tg}(\mathrm{Zp} 3-\mathrm{Cre}) 3 \mathrm{Mrt}$ (Zp3-Cre) transgene ${ }^{62}$ and to $B a x^{t m 1 S j k}$ mice. ${ }^{63}$ All mice were housed with free access to food and water and maintained on a 12- h:12- $\mathrm{h}$ lightdark cycle. All mouse experiments were performed in accordance with the Canadian Council on Animal Care (CCAC) guidelines for Use of Animals in Research and Laboratory Animal Care, under protocols approved by animal care committees of the Toronto Centre for Phenogenomics (TCP). To assess timing of excision, Zp3-Cre mice $^{62}$ were also crossed to reporter lines Tg(CAG-Bgeo/ALPP) $)^{1 \text { Lbe }}(\mathrm{Z} / \mathrm{AP})^{64}$ and Gt(ROSA)26Sor ${ }^{\text {tm9(CAG-tdTomato)Hze }}$ (tdTomato). ${ }^{65}$

Animals were genotyped for possession of either $\mathrm{MCl}^{-1^{+}}$or $\mathrm{MCl}-1^{\dagger}$ alleles using primers 5'-CTGAGAGTTGTACCGGACAA-3' (7MCL1) and 5'-GCAGTACAGGTTCA AGCCGATG-3' (6MCL1), and for Mcl-1 $1^{\text {null }}\left(\mathrm{MCl}^{-1}{ }^{-}\right)$allele using primers $7 \mathrm{MCL} 1$ and 5'-ACGCTCTTTAAGTGTTTGGCC-3' (2MCL1). Presence of Zp3-Cre transgene was assessed using 5'-TGATGAGGTTCGCAAGAACC-3' (CREF) and 5'-CCATGAGT GAACGAACCTGG-3' (CRER) and genotyping for $\mathrm{Bax}^{+}$and $\mathrm{Bax}^{-}$alleles utilized 5'-GAGCTGATCAGAACCATCATG-3' (BAX-EX5-F), 5'-GTTGACCAGAGTGGCG TAGG-3' (BAX-LN5-R) and 5'-CCGCTTCCATTGCTCAGCGG-3' (BAX-NEO).

Collection of oocytes and breeding. Immature (GV) oocytes were collected from antral follicles $46-48 \mathrm{~h}$ after pregnant mare's serum gonadotropin (PMSG; NHPP, Torrance, CA, USA or ProSpec, Rehovot, Israel (HOR-272)) priming and manually stripped of cumulus cells using narrow glass pipettes. For mature ovulated oocytes (MII), human chorionic gonadotropin (Sigma, Oakville, ON, Canada) priming was performed 44-48 $\mathrm{h}$ after PMSG and oocytes collected 14-16 h later; with cumulus removed by incubation in Hyaluronidase (Sigma). MII oocyte fragmentation rates were performed by MII culture in HTF media (Life Global, Guilford, CT, USA) supplemented with $0.1 \%$ BSA (Sigma) for $24 \mathrm{~h}$. For breeding rates, dams at 4-5 weeks were mated to young wild-type males for a 6 -month breeding trial and total litter number and size recorded. For $\mathrm{Mcl}-1 \mathrm{Cl}$ BaxDKO or BaxKO breedings, dams at 4-8 months of age were bred to wild-type 
$\left(\mathrm{Mcl}_{-1}{ }^{\mathrm{f} /+}\right)$ males for 2-3 months and litters were recorded. Immature human oocytes, obtained from patients undergoing IVF treatment, were donated to research after obtaining patient consent approved by the Research Ethics Board at Mount Sinai Hospital, Toronto. Details of gene expression studies are described below.

Real-time PCR with human oocytes. Obtained single human oocytes at GV stage free of cumulus cells were loaded into guanidine iso-thiocyanate solution and total nucleic acids were precipitated using glycogen as a carrier. ${ }^{22}$ Upon treatment with DNase (Sigma-Aldrich, St. Louis, MO, USA), reverse transcription was performed on the whole sample using Revert Aid Kit (Fermentas, Invitrogen Life Technologies, Burlington, ON, Canada) and oligo-dT primers. cDNA product was used in a real-time PCR reaction using LightCycler 480 SYBR Green I Master (Roche Applied Science, Indianapolis, IN, USA) and LightCycler 480 (Roche, Mannheim, Germany). The amplification profile included a pre-incubation step at $95^{\circ} \mathrm{C}$ for $5 \mathrm{~min}$, followed by denaturation at $95^{\circ} \mathrm{C}$, annealing at $62^{\circ} \mathrm{C}$ and extension at $72^{\circ} \mathrm{C}$. The target gene concentration for the oocyte samples was extrapolated utilizing the standard curve for each target and the data were expressed as relative ratio to $C_{P}$ value of $\beta$-ACTIN. Primer sequences for all genes analyzed (Supplementary Table S3).

Histological ovarian analyses. Ovaries from $\mathrm{Mcl}-1^{t /-}:$ Zp3-Cre $(\mathrm{Mcl}-$

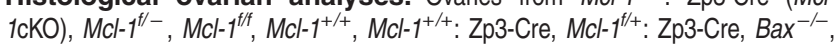

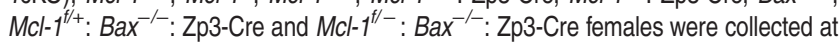
varying timepoints (6 months, 3 months, 3 weeks, PN14 or PN7) and fixed in Dietrichs (4\% formalin, $28 \%$ EtOH, 0.34N Glacial Acetic Acid (Sigma)) or 10\% formalin (Fisher, Ottawa, ON, Canada) and following standard dehydration protocols were embedded in paraffin wax and sectioned $(5 \mu \mathrm{m})$ using a LEICA (Concord, ON, Canada) RM2255 Microtome and then mounted on Superfrost plus slides. Sections fixed in Dietrichs were rehydrated and stained with a picric acid/methyl blue stain, allowing for better resolution for histomorphometric analyses. Every third section was counted for PN7 and PN14 ovaries, every fifth section counted for 3 weeks ovaries, and every 10th section for 3 months and 6 months. Oocytes with visible nuclei from primordial, primary, secondary and antral follicles were quantitated and recorded and multiplied by associated factor ( $x 3, x 5$ and $x 10)$ to gain an approximately full representation of the ovary. Atretic follicles were counted in sections from 3-week ovaries and are shown as a proportion of the total postsecondary growing follicles.

Ovaries from embryonic day 17.5 and PN3 tdTomato: Zp3-Cre animals were removed from animals and washed in MHTF. Entire tissue samples were viewed under LEICA DMI60003 Spinning Disc Confocal or LEICA MZ 165A Stereomicroscope using TRITC-Red laser (561 excitation, 620 emission), and imaged. Cryosections of ovaries from Z/AP: Zp3-Cre mice were postfixed in $0.2 \%$ glutaraldehyde and endogenous alkaline phosphatase (AP) activity was inactivated by heating for $30 \mathrm{~min}$ in PBS at $70^{\circ} \mathrm{C}$ and then rinsed in PBS. Sections were then washed in buffer (100 mM Tris-HCL pH 9.5, $100 \mathrm{mM} \mathrm{NaCl}, 10 \mathrm{mM} \mathrm{MgCl}_{2}$ ) for $10 \mathrm{~min}$ and stained with NBT/BCIP stain, washed in PTM $\left(0.1 \%\right.$ Tween20, $2 \mathrm{mM} \mathrm{MgCl}_{2}$, in PBS) and counterstained with Nuclear Fast Red.

Sections fixed in $10 \%$ formalin were rehydrated and used for immunohistochemical staining protocols. Sections were submitted to antigen retrieval at $\sim 95^{\circ} \mathrm{C}$ for $10 \mathrm{~min}$ in sodium citrate buffer (10 mM tri-sodium citrate (Sigma) pH 6.0 with $\mathrm{HCl}$ ), washed and blocked in 10\% normal horse serum (NHS) for $1 \mathrm{~h}$ before overnight incubation in primary antibody (in $10 \% \mathrm{NHS}$ ) at $4{ }^{\circ} \mathrm{C}$. Primary antibodies utilized include Mcl-1 (Rockland Immunochemicals, Limerick, PA, USA, 600-401-394S). Sections were then washed in PBS and incubated with secondary protocols from $A B C$ Vectastain kit (PK-4001; Vector Labs, Burlington, ON, Canada) and then visualized using diaminobenzidine (DAB) (Sigma) substrate. After time-sensitive stain development, sections were counterstained in hematoxylin (Sigma) for identification of cell nuclei.

TUNEL analyses. For detection of apoptotic cells, sections of PN1 ovaries were fixed in formalin, rehydrated and incubated in Proteinase $\mathrm{K}$ followed by permeabilization with $0.1 \%$ Triton-X. Slides were incubated in Reaction Mix ( $4 \mu \mathrm{M}$ biotin16-dUTP (Roche), $1.5 \mu \mathrm{M}$ dATP, 1X NEB4 buffer, 4 U/ul TdT enzyme (Roche) for $1.5 \mathrm{~h}$ at $37^{\circ} \mathrm{C}$ and incorporated nucleotides were detected with streptavidin $A B C$ Vectastain (Vector Labs) and visualized with DAB.

Mitochondrial analyses - live cell stains. MII ovulated oocytes of $\mathrm{Mcl}-1 \mathrm{cKO}$ and controls were collected and subjected to a number of assays for determination of mitochondrial function. Total and respiring mitochondria were stained using Mitotracker fluorescent dyes (MitoTracker Green FM (M7154), MitoTracker Red 580 (M22425); Molecular Probes, Invitrogen LifeTechnologies) added to HTF in $100 \mathrm{nM}$ concentration for $30 \mathrm{~min}$, then washed in $\mathrm{mHTF}$ and imaged. For total cellular levels of ROS, oocytes were incubated in $10 \mu \mathrm{M} \mathrm{2}$, $7^{\prime}$ dichlorofluorescein diacetate (DCFDA; Molecular Probes) or $5 \mu \mathrm{M}$ MitoSox (Molecular Probes) in HTF for $15 \mathrm{~min}$, washed in $\mathrm{mHTF}$ and imaged. The performance of fluorescent probes utilized for detection of ROS, and various mitochondrial markers was validated (Supplementary Figures S5D and E), using MII oocytes cultured in the presence of inducers of apoptosis or inhibitors of the electron transport chain. Induction of apoptosis in ovulated oocytes was done as previously described ${ }^{66}$ with $200 \mathrm{nM}$ doxorubicin (DXR; Alexis, Enzo LifeSciences, Farmingdale, NY, USA) for $20 \mathrm{~h}$ followed by incubation with MitoTracker Green, MitoTracker Red, DCFDA or MitoSox. For disruption of mitochondrial function and inhibition of electron transport chain, MIl oocytes were incubated in $100 \mathrm{nM}$ complex III inhibitor Antimycin (Sigma) or vehicle (ethanol) for $15 \mathrm{~min}$ followed by coincubation with mitochondrial dyes. Death rates for MII oocytes with antimycin treatment are included in Supplementary Figure S5E.

For mitochondrial membrane potential, MII oocytes were incubated with $5 \mu \mathrm{g} / \mathrm{ml}$ DePsipher (DePsipher, Trevigen (6300-100-K, Gaithersburg, MD, USA)) in HTF for $90 \mathrm{~min}$ then washed and imaged. The mitochondrial fluorescent distribution of each oocyte was visually separated into pattern 1 - oocytes with strong central green fluorescence distribution and peripheral scattering of polarized mitochondria (red); or pattern 2 - oocytes with diffuse distribution of green fluorescence and little to no peripheral polarized mitochondria (red).

To assess metabolic profile citrate, malate and fumarate levels, MII oocytes were first frozen on glass slides by dipping in cold isopentane. Oocytes were then freezedried and processed for metabolic profile using protocol delineated in Chi et al. ${ }^{67}$ ATP content in single oocytes was measured by Cell Titer GLO assay (Promega, Madison, WI, USA).

Immunofluorescence staining. GV or MII oocytes from $\mathrm{Mcl}-1 \mathrm{cKO}$ and control females were fixed in $10 \%$ formalin for $10 \mathrm{~min}$ and used for staining of markers of apoptosis and autophagy. Oocytes were first transferred to cooling $\sim 95^{\circ} \mathrm{C}$ sodium citrate buffer for antigen retrieval for $10 \mathrm{~min}$ using pulled-glass pipettes. Oocytes were moved to three washes in $0.1 \%$ Triton-X in $10 \mathrm{mM}$ PBS and then blocked in $10 \%$ NHS in PBS. Following this step, oocytes were incubated in primary antibody overnight at $4^{\circ} \mathrm{C}$. Primary antibodies used include anti-Beclin-1 (Santa Cruz Biotechnologies, Dallas, TX, USA, sc-11427), anti-LC-3 (MBL, EMD Millipore, Billerica, MA, USA, PM046), anti-Lamp1 (1D4B, Developmental Studies Hybridoma Bank, lowa City, IA, USA), anti-Lamp2 (ABL-93, Developmental Studies Hybridoma Bank), anti-Bax-NT (Upstate, EMD Millipore; 06-49), anti-BAX (Alexis, 210-003), anti-tubulin (Invitrogen LifeTechnologies, A11126), anti-actin (Santa Cruz Biotechnologies, sc-1616), anti-AIF (Santa Cruz Biotechnologies, sc-9416), antiMcl-1 (Santa Cruz Biotechnologies, sc-819) and anti-Mcl-1 (Rockland Immunochemicals, 600-401-394S). After primary antibody incubation, oocytes were transferred to $0.1 \% \mathrm{TX}$ washes and incubated in host-specific secondary antibody conjugated with Alexa Fluor dyes (Invitrogen LifeTechnologies) and counterstained with blue fluorescent 4',6-diamidino-2-phenylindole (DAPI, Sigma). Stained samples were mounted on slides in $50 \%$ glycerol for imaging. For negative controls, oocytes were exposed to nonspecific lgG or secondary antibody alone.

For evaluation of apoptotic induction, cytochrome c release, AIF release and pancaspase activity assays were performed on GV and MII oocytes with protocols modified from Carboxyfluorescein Multi-Caspase Activity Kit (Biomol, Enzo LifeSciences) and cytochrome c release kit (InnoCyte - Calbiochem, EMD MIllipore). A portion of denuded GV and MII oocytes were permeabilized for 10 min with digitonin buffer. Both permeabilized and non-permeabilized oocytes were then fixed, and processed through the staining. For AIF release, AIF (Santa Cruz Biotechnologies, sc-9416) was utilized instead of cytochrome c antibody. For pan-caspase activity, GV and MIl oocytes were incubated in FML-VAD-FMK stock dissolved in HTF medium for $2.5 \mathrm{~h}$ and washed and transferred to fixative as indicated in caspase activity kit. Oocytes were then washed and transferred to DAPI for 10-15 min, then mounted on Superfrost slides in 50\% glycerol and imaged, as mentioned previously. The efficacy of these markers of apoptotic induction was verified (Supplementary Figure S5D), using Mll oocytes cultured in the presence of $200 \mathrm{nM}$ DXR $^{66}$ for $14 \mathrm{~h}$ (Bax, cytochrome c) or $20 \mathrm{~h}$ (pan-caspase). MIl oocytes incubated with DXR for $24 \mathrm{~h}$ display death rates of $80 \%$.

Imaging. Stained oocytes were imaged using LEICA DMI60003 Spinning Disc Confocal microscope with appropriate filters (objectives: 10X/0.40NA, 20X/0.70NA). 
Images were acquired and analyzed using Volocity software (PerkinElmer, Waltham, MA, USA) with Z-stack images taken at $0.354 \mu \mathrm{m}$ increments across $10 \mu \mathrm{m}$ sections to the either side of the midpoint of the oocyte, and average mean fluorescent intensity obtained from each sample was used for comparative purposes and expressed as random fluorescent units (RFUs). Colocalization data images were analyzed using Imaris (Bitplane, Zurich, Switzerland) software.

Histological ovarian sections were imaged with a LEICA/LEITX DMRXE microscope (objectives: 5X/0.12 NA, 10X/0.30 NA, 20X/0.50 NA, 40X/0.85 NA, $63 \mathrm{X} / 0.75 \mathrm{NA}, 100 \mathrm{X} / 1.30 \mathrm{NA}$ ). Whole ovary images of TdTomato: Zp3-Cre mouse line and controls were acquired using the LEICA MZ16FA stereomicroscope (objectives: $4 \mathrm{X} / 0.45 \mathrm{NA}$ ), with appropriate filters.

Western blots. Two hundred GV oocytes from approximately 8-10 Mcl-1cKO and $\mathrm{MCl}^{-1}{ }^{+/+}$females were collected and lysed in RIPA buffer. Lysates were separated by SDS-PAGE and then transferred on to a PVDF membrane. Blots were incubated with anti-Mcl-1 (Rockland Immunochemicals, 600-401-394S) or anti-actin (Santa Cruz Biotechnologies, sc-1616). Membranes were washed and then incubated with specific HRP-conjugated donkey anti-rabbit (Santa Cruz Biotechnologies) or HRP-conjugated donkey anti-goat (Santa Cruz Biotechnologies) and detected using enhanced chemiluminescence (Thermo Scientific, Burlington, ON, Canada) SuperSignal West Femto Chemiluminescent Substrate and then imaged on the VersaDoc 5000MP Imaging System (Bio-Rad, Mississauga, ON, Canada).

Statistical analysis. Data were analyzed using either one-way ANOVA with Holm-Sidak multiple comparisons test (Breeding performance, histomorphometric analyses, PCC, PCC-C); or using non-parametric Kruskal-Wallis one-way ANOVA on ranks, followed by Dunns post-hoc test for comparisons between groups, when normality failed or sample sizes were vastly different (ovulation rates, active Bax (GV), beclin, LC-3, MitoTracker green and red, active Bax (MII), fragmentation rates, MCC-A, MCC-B, MitoSox, ROS, cytochrome c, caspase activity, chromosomal misalignments). Statistical measures for two groups were performed using the unpaired $t$-test (histomorphometric analyses, atresia rates, metabolites), two-way ANOVA (DePsipher) or $\chi^{2}$ test (chromosome misalignments; Figure 6). Association maternal age and gene expression was done by Pearson correlation. All analysis was done using the Sigma Stat (Systat, San Jose, CA, USA) or PRISM software package (Graph Pad, San Diego, CA, USA). In all cases, differences were considered significant if value reached $P<0.05$.

\section{Conflict of Interest}

The authors declare no conflict of interest.

Acknowledgements. We acknowledge S Cao, A Borrego-Alvarez and T Yavorska for their technical assistance. We also express our thanks to the staff of the Reproductive Biology Unit at MSH as well as patients for donations of immature oocytes. This work was supported by operating grant from Canadian Institutes of Health Research (FRN 123518) and Canada Research Chair CRC/CFI program (AJ).

1. Forabosco A, Sforza C. Establishment of ovarian reserve: a quantitative morphometric study of the developing human ovary. Fertil Steril 2007; 88: 675-683.

2. Wallace WH, Kelsey TW. Human ovarian reserve from conception to the menopause. PloS One 2010; 5: e8772.

3. Morita Y, Tilly JL. Oocyte apoptosis: like sand through an hourglass. Dev Biol 1999; 213 $1-17$.

4. Conway GS. Premature ovarian failure. Curr Opin Obstet Gynecol 1997; 9: 202-206.

5. Goswami D, Conway GS. Premature ovarian failure. Horm Res 2007; 68: 196-202.

6. Faddy MJ, Gosden RG. A model conforming the decline in follicle numbers to the age of menopause in women. Hum Reprod 1996; 11: 1484-1486.

7. Faddy MJ. Follicle dynamics during ovarian ageing. Mol Cell Endocrinol 2000; 163: 43-48.

8. Hamatani T, Falco G, Carter MG, Akutsu H, Stagg CA, Sharov AA et al. Age-associated alteration of gene expression patterns in mouse oocytes. Hum Mol Genet 2004; 13: 2263-2278.

9. Hunt PA, Hassold TJ. Human female meiosis: what makes a good egg go bad? Trends Genet 2008; 24: 86-93.

10. Jurisicova A, Rogers I, Fasciani A, Casper RF, Varmuza S. Effect of maternal age and conditions of fertilization on programmed cell death during murine preimplantation embryo development. Mol Hum Reprod 1998; 4: 139-145.

11. Ghafari F, Gutierrez CG, Hartshorne GM. Apoptosis in mouse fetal and neonatal oocytes during meiotic prophase one. BMC Dev Biol 2007; $7: 87$.
12. Kerr JB, Myers $M$, Anderson RA. The dynamics of the primordial follicle reserve. Reproduction 2013; 146: R205-R215.

13. Escobar ML, Echeverria OM, Ortiz R, Vazquez-Nin GH. Combined apoptosis and autophagy, the process that eliminates the oocytes of atretic follicles in immature rats. Apoptosis 2008; 13: $1253-1266$

14. Lobascio AM, Klinger FG, Scaldaferri ML, Farini D, De Felici M. Analysis of programmed cell death in mouse fetal oocytes. Reproduction 2007; 134: 241-252.

15. De Felici M, Lobascio AM, Klinger FG. Cell death in fetal oocytes: many players for multiple pathways. Autophagy 2008; 4: 240-242.

16. Jones RL, Pepling ME. Role of the antiapoptotic proteins BCL2 and MCL1 in the neonatal mouse ovary. Biol Reprod 2013; 88: 46.

17. Print CG, Loveland KL, Gibson L, Meehan T, Stylianou A, Wreford N et al. Apoptosis regulator bcl-w is essential for spermatogenesis but appears otherwise redundant. Proc Natl Acad Sci USA 1998; 95: 12424-12431.

18. Ratts VS, Flaws JA, Kolp R, Sorenson CM, Tilly JL. Ablation of bcl-2 gene expression decreases the numbers of oocytes and primordial follicles established in the post-natal female mouse gonad. Endocrinology 1995; 136: 3665-3668.

19. Russell HR, Lee Y, Miller HL, Zhao J, McKinnon PJ. Murine ovarian development is not affected by inactivation of the bcl-2 family member diva. Mol Cell Biol 2002; 22: 6866-6870.

20. Rucker EB 3rd, Dierisseau P, Wagner KU, Garrett L, Wynshaw-Boris A, Flaws JA et al. $B c l-x$ and Bax regulate mouse primordial germ cell survival and apoptosis during embryogenesis. Mol Endocrinol 2000; 14: 1038-1052.

21. Riedlinger G, Okagaki R, Wagner KU, Rucker EB 3rd, Oka T, Miyoshi K et al. Bcl-x is not required for maintenance of follicles and corpus luteum in the postnatal mouse ovary. Biol Reprod 2002; 66: 438-444.

22. Jurisicova A, Latham KE, Casper RF, Varmuza SL. Expression and regulation of genes associated with cell death during murine preimplantation embryo development. Mol Reprod Dev 1998; 51: 243-253.

23. Tilly JL. Commuting the death sentence: how oocytes strive to survive. Nat Rev Mol Cell Biol 2001; 2: 838-848.

24. Hartley PS, Bayne RA, Robinson LL, Fulton N, Anderson RA. Developmental changes in expression of myeloid cell leukemia-1 in human germ cells during oogenesis and early folliculogenesis. J Clin Eendocrinol Metab 2002; 87: 3417-3427.

25. Jurisicova A, Acton BM. Deadly decisions: the role of genes regulating programmed cell death in human preimplantation embryo development. Reproduction 2004; 128: 281-291.

26. Youle RJ, Strasser A. The BCL-2 protein family: opposing activities that mediate cell death. Nat Rev Mol Cell Biol 2008; 9: 47-59.

27. Erlich S, Mizrachy L, Segev O, Lindenboim L, Zmira O, Adi-Harel S et al. Differential interactions between Beclin 1 and Bcl-2 family members. Autophagy 2007; 3: 561-568.

28. Germain M, Nguyen AP, Le Grand JN, Arbour N, Vanderluit JL, Park DS et al. MCL-1 is a stress sensor that regulates autophagy in a developmentally regulated manner. EMBO J 2011; 30: 395-407.

29. Kang R, Zeh HJ, Lotze MT, Tang D. The Beclin 1 network regulates autophagy and apoptosis. Cell Death Differ 2011; 18: 571-580.

30. Kirisako T, Baba M, Ishihara N, Miyazawa K, Ohsumi M, Yoshimori T et al. Formation process of autophagosome is traced with Apg8/Aut7p in yeast. J Cell Biol 1999; 147: 435-446.

31. Huynh KK, Eskelinen EL, Scott CC, Malevanets A, Saftig P, Grinstein S. LAMP proteins are required for fusion of lysosomes with phagosomes. EMBO J 2007; 26: 313-324.

32. Wai T, Ao A, Zhang X, Cyr D, Dufort D, Shoubridge EA. The role of mitochondrial DNA copy number in mammalian fertility. Biol Reprod 2010; 83: 52-62.

33. Zeng HT, Ren Z, Yeung WS, Shu YM, Xu YW, Zhuang GL et al. Low mitochondrial DNA and ATP contents contribute to the absence of birefringent spindle imaged with PolScope in in vitro matured human oocytes. Hum Reprod 2007; 22: 1681-1686.

34. Downs SM, Humpherson PG, Leese HJ. Pyruvate utilization by mouse oocytes is influenced by meiotic status and the cumulus oophorus. Mol Reprod Dev 2002; 62: 113-123.

35. Perciavalle RM, Stewart DP, Koss B, Lynch J, Milasta S, Bathina M et al. Anti-apoptotic MCL-1 localizes to the mitochondrial matrix and couples mitochondrial fusion to respiration. Nat Cell Biol 2012; 14: 575-583.

36. Wang X, Bathina M, Lynch J, Koss B, Calabrese C, Frase S et al. Deletion of MCL-1 causes lethal cardiac failure and mitochondrial dysfunction. Genes Dev 2013; 27: 1351-1364.

37. Boveris A, Cadenas E, Stoppani AO. Role of ubiquinone in the mitochondrial generation of hydrogen peroxide. Biochem J 1976; 156: 435-444.

38. Turrens JF, Alexandre A, Lehninger AL. Ubisemiquinone is the electron donor for superoxide formation by complex III of heart mitochondria. Arch Biochem Biophys 1985; 237: 408-414.

39. Thouas GA, Trounson AO, Jones GM. Developmental effects of sublethal mitochondrial injury in mouse oocytes. Biol Reprod 2006; 74: 969-977.

40. Thouas GA, Trounson AO, Wolvetang EJ, Jones GM. Mitochondrial dysfunction in mouse oocytes results in preimplantation embryo arrest in vitro. Biol Reprod 2004; 71: 1936-1942.

41. Johnson MT, Freeman EA, Gardner DK, Hunt PA. Oxidative metabolism of pyruvate is required for meiotic maturation of murine oocytes in vivo. Biol Reprod 2007; 77: 2-8.

42. Norris RP, Freudzon M, Mehlmann LM, Cowan AE, Simon AM, Paul DL et al. Luteinizing hormone causes MAP kinase-dependent phosphorylation and closure of connexin 43 gap junctions in mouse ovarian follicles: one of two paths to meiotic resumption. Development 2008; 135: 3229-3238

43. Perez Gl, Tao XJ, Tilly JL. Fragmentation and death (a.k.a. apoptosis) of ovulated oocytes. Mol Hum Reprod 1999; 5: 414-420. 
44. Stallock J, Molyneaux K, Schaible K, Knudson CM, Wylie C. The pro-apoptotic gene Bax is required for the death of ectopic primordial germ cells during their migration in the mouse embryo. Development 2003; 130: 6589-6597.

45. Greenfeld CR, Pepling ME, Babus JK, Furth PA, Flaws JA. BAX regulates follicular endowment in mice. Reproduction 2007; 133: 865-876.

46. Perez Gl, Jurisicova A, Wise L, Lipina T, Kanisek M, Bechard A et al. Absence of the proapoptotic Bax protein extends fertility and alleviates age-related health complications in female mice. Proc Natl Acad Sci USA 2007; 104: 5229-5234.

47. Opferman JT, Letai A, Beard C, Sorcinelli MD, Ong CC, Korsmeyer SJ. Development and maintenance of B and T lymphocytes requires antiapoptotic MCL-1. Nature 2003; 426 : 671-676.

48. Opferman JT, Iwasaki H, Ong CC, Suh H, Mizuno S, Akashi K et al. Obligate role of antiapoptotic MCL-1 in the survival of hematopoietic stem cells. Science 2005; 307: 1101-1104.

49. Jurisicova A, Lee HJ, D'Estaing SG, Tilly J, Perez Gl. Molecular requirements for doxorubicin-mediated death in murine oocytes. Cell Death Differ 2006; 13: 1466-1474.

50. Lefevre B, Gougeon A, Nome F, Testart J. In vivo changes in oocyte germinal vesicle related to follicular quality and size at mid-follicular phase during stimulated cycles in the cynomolgus monkey. Reprod Nutr Dev 1989; 29: 523-531.

51. Conti M, Hsieh M, Zamah AM, Oh JS. Novel signaling mechanisms in the ovary during oocyte maturation and ovulation. Mol Cell Endocrinol 2012; 356: 65-73.

52. Norris RP, Ratzan WJ, Freudzon M, Mehlmann LM, Krall J, Movsesian MA et al. Cyclic GMP from the surrounding somatic cells regulates cyclic AMP and meiosis in the mouse oocyte. Development 2009; 136: 1869-1878.

53. Colonna R, Mangia F. Mechanisms of amino acid uptake in cumulus-enclosed mouse oocytes. Biol Reprod 1983; 28: 797-803.

54. Eppig JJ, Pendola FL, Wigglesworth K, Pendola JK. Mouse oocytes regulate metabolic cooperativity between granulosa cells and oocytes: amino acid transport. Biol Reprod 2005; 73: 351-357.

55. Eppig JJ. Analysis of mouse oogenesis in vitro. Oocyte isolation and the utilization of exogenous energy sources by growing oocytes. J Exp Zool 1976; 198: 375-382.

56. Biggers JD, Whittingham DG, Donahue RP. The pattern of energy metabolism in the mouse oocyte and zygote. Proc Natl Acad Sci USA 1967; 58: 560-567.

57. Schutt F, Aretz S, Auffarth GU, Kopitz J. Moderately reduced ATP levels promote oxidative stress and debilitate autophagic and phagocytic capacities in human RPE cells. Invest Ophthalmol Vis Sci 2012; 53: 5354-5361.

58. Schellens JP, Meijer AJ. Energy depletion and autophagy. Cytochemical and biochemical studies in isolated rat hepatocytes. Histochem J 1991; 23: 460-466.
59. Hoppins S, Edlich F, Cleland MM, Banerjee S, McCaffery JM, Youle RJ et al. The soluble form of Bax regulates mitochondrial fusion via MFN2 homotypic complexes. Mol Cell 2011; 41: $150-160$.

60. Rinkenberger JL, Horning S, Klocke B, Roth K, Korsmeyer SJ. Mcl-1 deficiency results in peri-implantation embryonic lethality. Genes Dev 2000; 14: 23-27.

61. Tarin JJ, Perez-Albala S, Cano A. Cellular and morphological traits of oocytes retrieved from aging mice after exogenous ovarian stimulation. Biol Reprod 2001; 65 $141-150$.

62. Lewandoski M, Wassarman KM, Martin GR. Zp3-cre, a transgenic mouse line for the activation or inactivation of loxP-flanked target genes specifically in the female germ line. Curr Biol 1997; 7: 148-151.

63. Knudson CM, Tung KS, Tourtellotte WG, Brown GA, Korsmeyer SJ. Bax-deficient mice with lymphoid hyperplasia and male germ cell death. Science 1995; 270: 96-99.

64. Lobe CG, Koop KE, Kreppner W, Lomeli H, Gertsenstein M, Nagy A. Z/AP, a double reporter for cre-mediated recombination. Dev Biol 1999; 208: 281-292.

65. Madisen L, Zwingman TA, Sunkin SM, Oh SW, Zariwala HA, Gu H et al. A robust and highthroughput Cre reporting and characterization system for the whole mouse brain. Nat Neurosci 2010; 13: 133-140.

66. Jurisicova A, Lee HJ, D'Estaing SG, Tilly J, Perez GI. Molecular requirements for doxorubicin-mediated death in murine oocytes. Cell Death Differ 2006; 13: 1466-1474.

67. Chi MM, Hoehn A, Moley KH. Metabolic changes in the glucose-induced apoptotic blastocyst suggest alterations in mitochondrial physiology. Am J Physiol Endocrinol Metab 2002; 283 E226-E232.

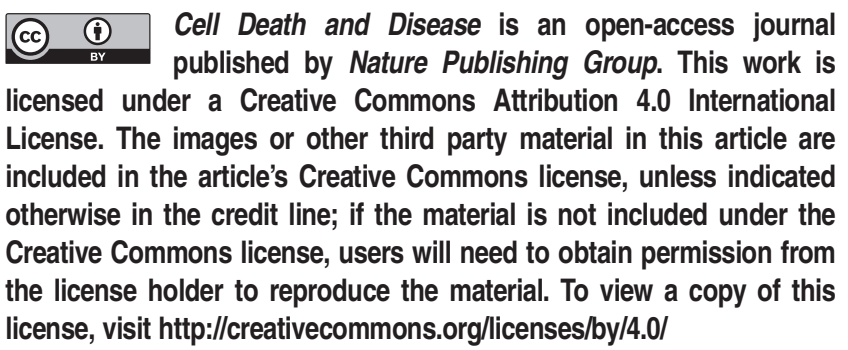

Supplementary Information accompanies this paper on Cell Death and Disease website (http://www.nature.com/cddis) 